\title{
Eigenvalue monotonicity of $q$-Laplacians of trees along a poset
}

\author{
Mukesh Kumar Nagar \\ Department of Mathematics \\ Indian Institute of Technology, Bombay \\ Mumbai 400 076, India. \\ email: mukesh.kr.nagar@gmail.com
}

October 7, 2018

\begin{abstract}
Let $T$ be a tree on $n$ vertices with $q$-Laplacian $\mathcal{L}_{T}^{q}$. Let $\mathrm{GTS}_{n}$ be the generalized tree shift poset on the set of unlabelled trees with $n$ vertices. We prove that for all $q \in \mathbb{R}$, going up on $\mathrm{GTS}_{n}$ has the following effect: the spectral radius and the second smallest eigenvalue of $\mathcal{L}_{T}^{q}$ increase while the smallest eigenvalue of $\mathcal{L}_{T}^{q}$ decreases. These generalize known results for eigenvalues of the Laplacian. As a corollary, we obtain consequences about the eigenvalues of $q, t$-Laplacians and exponential distance matrices of trees.
\end{abstract}

\section{Introduction}

In [10], Kelmans defined an operation on graphs called the Kelmans' transformation. This transformation has nice properties: it increases the spectral radius of adjacency matrix (see Csikvári [4]) and decreases the number of spanning trees (see Satyanarayana, Schoppman and Suffel [13]).

Motivated by Kelmans' transformation, Csikvári [5, 6] defined a poset on the set of unlabelled trees with $n$ vertices denoted here as $\mathrm{GTS}_{n}$ (see Subsection 2.1 for the definition). Among other results, he proved the following.

Theorem 1 (Csikvári) Going up on $\mathrm{GTS}_{n}$ increases both the algebraic connectivity (the second smallest eigenvalue) and the largest eigenvalue of the Laplacian matrix of trees.

For a graph $G$, its Laplacian matrix $L_{G}$ has a $q$-analogue, denoted by $\mathcal{L}_{G}^{q}$ called the $q$-Laplacian of $G$. The entries of $\mathcal{L}_{G}^{q}$ are polynomials in a real variable $q$. The matrix $\mathcal{L}_{G}^{q}$ is defined as $\mathcal{L}_{G}^{q}=$ $I+q^{2}(D-I)-q A$, where $D$ is the diagonal matrix with degrees on the diagonal and $A$ is the adjacency matrix of $G$. Clearly when $q=1, \mathcal{L}_{G}^{q}=L_{G}$. The matrix $\mathcal{L}_{G}^{q}$ has connections with the 
Ihara-Selberg zeta function of a graph (see Bass [3] and Foata and Zeilberger [7]). When $G$ is a tree $T, \mathcal{L}_{T}^{q}$ has connections with distance matrix (see Bapat, Lal and Pati [1]).

In this paper, we prove the following more general result about the eigenvalues of $\mathcal{L}_{T}^{q}$. For a fixed $q \in \mathbb{R}$, let $\lambda_{\max }\left(\mathcal{L}_{T}^{q}\right), \lambda_{\min }\left(\mathcal{L}_{T}^{q}\right)$ and $\lambda_{a}\left(\mathcal{L}_{T}^{q}\right)$ be the largest, the smallest and the second smallest eigenvalues of $\mathcal{L}_{T}^{q}$ respectively.

Theorem 2 Let $T_{1}$ and $T_{2}$ be two trees with $n$ vertices such that $T_{1} \leq{ }_{\mathrm{GTS}} T_{2}$ on $\mathrm{GTS}_{n}$. Let $\mathcal{L}_{T_{1}}^{q}$ and $\mathcal{L}_{T_{2}}^{q}$ be the $q$-Laplacians of $T_{1}$ and $T_{2}$ respectively. Then, for all $q \in \mathbb{R}$, we have

$$
\lambda_{\max }\left(\mathcal{L}_{T_{1}}^{q}\right) \leq \lambda_{\max }\left(\mathcal{L}_{T_{2}}^{q}\right), \lambda_{a}\left(\mathcal{L}_{T_{1}}^{q}\right) \leq \lambda_{a}\left(\mathcal{L}_{T_{2}}^{q}\right) \text { and } \lambda_{\min }\left(\mathcal{L}_{T_{1}}^{q}\right) \geq \lambda_{\min }\left(\mathcal{L}_{T_{2}}^{q}\right)
$$

Thus for all $q \in \mathbb{R}$, three eigenvalues of $\mathcal{L}_{T}^{q}$ exhibit monotonicity when we go up on $\mathrm{GTS}_{n}$. It is easy to see that Theorem 2 gives us Theorem 1 by setting $q=1$. Theorem 2 gives us one extra result which is trivial when $q=1$, as it is well known that the smallest eigenvalue of the Laplacian $L_{G}$ of a graph $G$ is zero. Thus it is constant on $\mathrm{GTS}_{n}$.

Note that when $q=0, \mathcal{L}_{T}^{q}$ is the identity matrix for all trees $T$. In this case, all the eigenvalues of $\mathcal{L}_{T}^{q}$ on $\mathrm{GTS}_{n}$ are constant and hence Theorem 2 is trivially true. Thus in this work, we will assume $q \neq 0$.

The Laplacian $L_{G}$ has a bivariate analogue denoted by $\mathcal{L}_{G}^{q, t}$ called the $q$, $t$-Laplacian of $G$ (see Section 7 for the definition). $\mathcal{L}_{G}^{q, t}$ was defined by Bapat and Sivasubramanian in [2] to get a bivariate version of the Ihara-Selberg zeta function of $G$. When the graph $G$ is a tree $T, \mathcal{L}_{T}^{q, t}$ has connections with bivariate versions of distance matrices (see [2]). Here, both $q$ and $t$ are variables and we will let them take both real and complex values. Our results have implication for the eigenvalues of $\mathcal{L}_{T}^{q, t}$ for some values of $q, t \in \mathbb{C}$.

This paper is organized as follows: Section 2 describes some preliminaries on the poset $\mathrm{GTS}_{n}$ and exponential distance matrix $\mathrm{ED}_{T}$ of a tree $T$. In Section 3, we extend the general lemma proved by Csikvári [6] to the characteristic polynomial of $\mathcal{L}_{T}^{q}$. In Section 4, we prove an interlacing results about the eigenvalues of $\mathcal{L}_{T}^{q}$ for all $q \in \mathbb{R}$. In Section 5, inspired by Csikvári [6], we define and prove some properties of an auxiliary bivariate polynomial which in Section 6 is used to prove Theorem 2. Theorem 2 can be used to obtain upper bounds on the largest and the second smallest eigenvalues of $\mathcal{L}_{T}^{q}$ for all $q \in \mathbb{R}$, see Corollaries 28 and 34 respectively. Theorem 2 also has consequences for eigenvalues of the $q, t$-Laplacian and the exponential distance matrices of trees. These are obtained in Sections 7 and 8 respectively.

\section{Preliminaries}

In the first part we give some preliminaries on the poset $\mathrm{GTS}_{n}$ and in the second subsection we cover some preliminary results of the $q$-Laplacian matrix $\mathcal{L}_{T}^{q}$ and of the exponential distance matrix $\mathrm{ED}_{T}$ of a tree $T$. 


\subsection{The Poset GTS}

We recall the definition of the generalized tree shift poset $\mathrm{GTS}_{n}$ defined by Csikvári [5]. Let $P_{n}$ and $S_{n}$ denote the path tree and the star tree on $n$ vertices respectively.

Definition 3 Let $T_{1}$ be a tree on $n$ vertices. Let $P_{k}$ be a path between two vertices in $T_{1}$, say 1 and $k$ such that each internal vertex (if it exists) of $P_{k}$ has degree 2. Let $k-1$ be the neighbour of $k$ on $P_{k}$. Construct a new tree $T_{2}$ from $T_{1}$ by moving all the neighbours of $k$ other than $k-1$ to the vertex 1 . This operation is called a generalized tree shift. This is illustrated in Figure 1
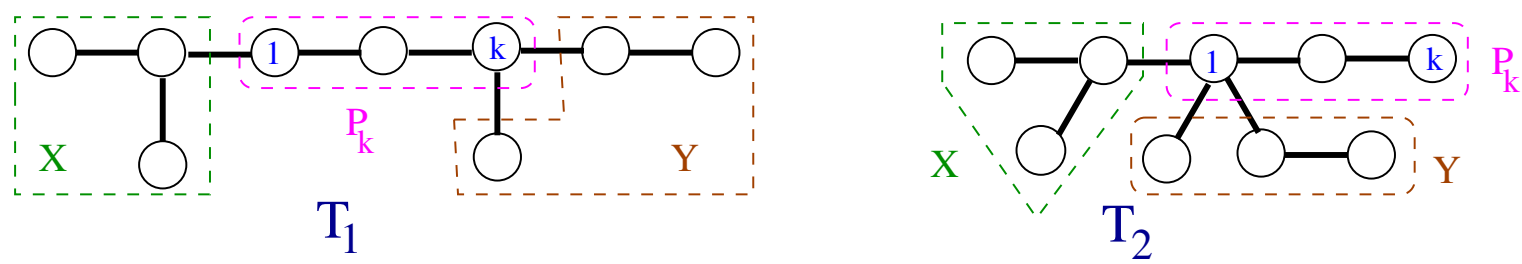

Figure 1: Example of $T_{1} \leq_{\mathrm{GTS}_{n}} T_{2}$.

The generalized tree shift operation gives us a partial order on the set of unlabelled trees with $n$ vertices, denoted by " $\leq_{\mathrm{GTS}_{n}}$ ". If $T_{1} \leq_{\mathrm{GTS}_{n}} T_{2}$ and there is no tree $T$ with $T \neq T_{1}, T_{2}$ such that $T_{1} \leq_{\mathrm{GTS}_{n}} T \leq_{\mathrm{GTS}_{n}} T_{2}$, then we say $T_{2}$ covers $T_{1}$ in $\mathrm{GTS}_{n}$. If either 1 or $k$ is a leaf vertex in $T_{1}$ then it is simple to check that $T_{2}$ is isomorphic to $T_{1}$. If neither vertex 1 nor vertex $k$ is a leaf in $T_{1}$ then $T_{2}$ covers $T_{1}$. In this case, the number of leaf vertices in $T_{2}$ is one more than the number of leaf vertices in $T_{1}$. Conversely, if $T_{2}$ covers $T_{1}$ then there exists vertices $1, k$ which witnesses the covering relation. We will use the vertices $1, k$ only for this purpose in this paper. We refer the reader to Csikvári [6] for Hasse diagram of GTS ${ }_{6}$. Csikvári [5, Theorem 2.4 and Corollary 2.5] proved the following result.

Lemma 4 (Csikvári) Let $T$ be a tree with $n$ vertices different from $P_{n}$. Then, there exists $T^{\prime}$ such that $T^{\prime} \leq_{\mathrm{GTS}_{n}} T$. Let $T$ be a tree with $n$ vertices different from $S_{n}$. Then, there exists $T^{\prime \prime}$ such that $T \leq_{\mathrm{GTS}_{n}} T^{\prime \prime}$. Moreover, $P_{n}$ and $S_{n}$ are the only minimal and the maximal elements of $\mathrm{GTS}_{n}$ respectively.

Thus monotonicity results on $\mathrm{GTS}_{n}$ show that max-min pair is either $\left(P_{n}, S_{n}\right)$ or $\left(S_{n}, P_{n}\right)$.

\section{2 $q$-Laplacian and Exponential distance matrix of a tree}

For a graph $G$, let $B_{1}=\left.\mathcal{L}_{G}^{q}\right|_{q=1}=L_{G}=D-A$ and $B_{2}=\left.\mathcal{L}_{G}^{q}\right|_{q=-1}=D+A$, where $D$ is the diagonal matrix with degrees on the diagonal and $A$ is the adjacency matrix of $G$. It is well known that $B_{1}$ and $B_{2}$ are similar matrices for a bipartite graph $G$. Thus, when $q= \pm 1$, the $q$ Laplacian matrix $\mathcal{L}_{T}^{q}$ of a tree $T$ is positive semidefinite. In this case, all the eigenvalues of $\mathcal{L}_{T}^{q}$ are non-negative and the multiplicity of zero as an eigenvalue of $\mathcal{L}_{T}^{q}$ is 1. Bapat, Lal and Pati [1, Propositions 3.4 and 3.7] proved the following result. 
Lemma 5 (Bapat, Lal and Pati) Let $T$ be a tree on $n$ vertices with $q$-Laplacian $\mathcal{L}_{T}^{q}$. Then,

1. $\operatorname{det}\left(\mathcal{L}_{T}^{q}\right)=1-q^{2}$.

2. $\mathcal{L}_{T}^{q}$ is positive definite when $q \in \mathbb{R}$ with $|q|<1$.

3. $\mathcal{L}_{T}^{q}$ has exactly one negative eigenvalue when $q \in \mathbb{R}$ with $|q|>1$.

In [1], Bapat, Lal and Pati introduced the exponential distance matrix $\mathrm{ED}_{T}$ of a tree $T$. We recall its definition, let $T$ be a tree with $n$ vertices, then its exponential distance matrix $\mathrm{ED}_{T}=$ $\left(e_{i, j}\right)_{1 \leq i, j \leq n}$ is defined as follows: the entry $e_{i, j}=1$, if $i=j$ and $e_{i, j}=q^{d_{i, j}}$, if $i \neq j$, where $d_{i, j}$ is the distance between vertex $i$ and vertex $j$ in $T$. Clearly $\mathrm{ED}_{T}$ is a symmetric matrix, hence all its eigenvalues are real. Bapat, Lal and Pati [1, Lemma 3.8] proved the following result about the relationship between the eigenvalues of $\mathcal{L}_{T}^{q}$ and $\mathrm{ED}_{T}$.

Lemma 6 (Bapat, Lal and Pati) Let $T$ be a tree with $n$ vertices. Let $\mathcal{L}_{T}^{q}$ and $\mathrm{ED}_{T}$ be the $q$ Laplacian and the exponential distance matrix of $T$ respectively. If $q \neq \pm 1$, then $\mathrm{ED}_{T}^{-1}=\frac{1}{1-q^{2}} \mathcal{L}_{T}^{q}$. Moreover, $\frac{1-q^{2}}{\lambda_{i}\left(\mathcal{L}_{T}^{q}\right)}$ is an eigenvalue of $\mathrm{ED}_{T}$, where $\lambda_{i}\left(\mathcal{L}_{T}^{q}\right)$ is an eigenvalue of $\mathcal{L}_{T}^{q}$.

Let $T$ be a tree on $n$ vertices with $q$-Laplacian $\mathcal{L}_{T}^{q}$ and exponential distance matrix $\mathrm{ED}_{T}$. Let the eigenvalues of $\mathcal{L}_{T}^{q}$ be $\lambda_{\max }\left(\mathcal{L}_{T}^{q}\right)=\lambda_{1}\left(\mathcal{L}_{T}^{q}\right) \geq \lambda_{2}\left(\mathcal{L}_{T}^{q}\right) \geq \cdots \geq \lambda_{n}\left(\mathcal{L}_{T}^{q}\right)=\lambda_{\min }\left(\mathcal{L}_{T}^{q}\right)$. From Lemma 5 , it is easy to see that $\lambda_{n-1}\left(\mathcal{L}_{T}^{q}\right)>0$ for all $q \in \mathbb{R}$. Let the eigenvalues of $\operatorname{ED}_{T}$ be $\lambda_{\max }\left(\mathrm{ED}_{T}\right)=$ $\lambda_{1}\left(E_{T}\right) \geq \lambda_{2}\left(E_{T}\right) \geq \cdots \geq \lambda_{n}\left(E_{T}\right)=\lambda_{\min }\left(E_{T}\right)$. Nagar and Sivasubramanian [12, Remark 8 and Corollary 11] proved that both $\left.\mathcal{L}_{T}^{q}\right|_{q=\alpha}$ and $\left.\mathcal{L}_{T}^{q}\right|_{q=-\alpha}$ have the same characteristic polynomial for a tree $T$ and for all $\alpha \in \mathbb{R}$. Thus, the multiset of eigenvalues of both the matrices $\left.\mathcal{L}_{T}^{q}\right|_{q=\alpha}$ and $\left.\mathcal{L}_{T}^{q}\right|_{q=-\alpha}$ are equal. This argument is used to prove the following lemma which will be used in the proof of Lemma 32 .

Lemma 7 Let $T$ be a tree on $n$ vertices with $q$-Laplacian $\mathcal{L}_{T}^{q}$. Then for all $q \in \mathbb{R} \backslash\{0\}$, the algebraic multiplicity of $\lambda_{\min }\left(\mathcal{L}_{T}^{q}\right)$ as an eigenvalue of $\mathcal{L}_{T}^{q}$ is 1 .

Proof: As for all $\alpha \in \mathbb{R}$, the multiset of eigenvalues of both the matrices $\left.\mathcal{L}_{T}^{q}\right|_{q=\alpha}$ and $\left.\mathcal{L}_{T}^{q}\right|_{q=-\alpha}$ are equal, it is sufficient to prove the corollary when $q \in \mathbb{R}$ with $q>0$. We first consider the case when $q \in \mathbb{R}$ with $q \geq 1$, from Lemma 5 , $\lambda_{\text {min }}\left(\mathcal{L}_{T}^{q}\right) \leq 0$ and $\lambda_{n-1}\left(\mathcal{L}_{T}^{q}\right)>0$. Thus, when $q \in \mathbb{R}$ with $q \geq 1$, the algebraic multiplicity of $\lambda_{\min }\left(\mathcal{L}_{T}^{q}\right)$ as an eigenvalue of $\mathcal{L}_{T}^{q}$ is 1 .

We next consider the case when $q \in \mathbb{R}$ with $0<q<1$. In this case each entry of the matrix $\mathrm{ED}_{T}$ is strictly positive. Therefore by Perron's Theorem (see Horn and Johnson [9, page 526 ]), the algebraic multiplicity of $\lambda_{\max }\left(E_{T}\right)$ as an eigenvalue of $E_{T}$ is 1 for all $q \in \mathbb{R}$ with $0<q<1$. In this case, from Lemma 6, we get the following.

$$
\lambda_{\max }\left(\mathrm{ED}_{T}\right)=\frac{1-q^{2}}{\lambda_{\min }\left(\mathcal{L}_{T}^{q}\right)}>\frac{1-q^{2}}{\lambda_{n-1}\left(\mathcal{L}_{T}^{q}\right)}=\lambda_{2}\left(\mathrm{ED}_{T}\right)
$$

Thus, $\lambda_{\min }\left(\mathcal{L}_{T}^{q}\right)<\lambda_{n-1}\left(\mathcal{L}_{T}^{q}\right)$ for all $q \in \mathbb{R}$ with $0<q<1$ and the proof is complete.

Remark 8 Lemma 7 generalizes the known theorem (see Godsil and Royle [8]) that $\lambda_{2}\left(L_{G}\right)>0$ for a connected graph $G$ as follows: The algebraic multiplicity of $\lambda_{\min }\left(L_{G}\right)=0$ is 1 . Lemma 7 shows that for a tree $T$, the algebraic multiplicity of $\lambda_{\min }\left(\mathcal{L}_{T}^{q}\right)$ for all $q \in \mathbb{R} \backslash\{0\}$ is again 1 . It would be interesting to see if $\lambda_{2}\left(\mathcal{L}_{G}^{q}\right) \neq \lambda_{1}\left(\mathcal{L}_{G}^{q}\right)$ for all $q \in \mathbb{R} \backslash\{0\}$ for all connected graphs $G$. 


\section{General lemma}

We begin by recalling the following definition due to Csikvári [6]. Let $Q_{1}=\left(V_{1}, E_{1}\right)$ and $Q_{2}=$ $\left(V_{2}, E_{2}\right)$ be two trees on disjoint vertex sets. Let $v_{1} \in V_{1}$ and $v_{2} \in V_{2}$. Construct a new tree $T$ by moving all the neighbours of $v_{2}$ to $v_{1}$ and then deleting $v_{2}$. We thus treat $v_{1}$ and $v_{2}$ as a single vertex in $T$. The obtained tree $T$ is denoted by $Q_{1}^{v_{1}}: Q_{2}^{v_{2}}$ and has vertex set $V\left(Q_{1}^{v_{1}}: Q_{2}^{v_{2}}\right)=V_{1} \cup V_{2}-\left\{v_{2}\right\}$ and edge set $E\left(Q_{1}^{v_{1}}: Q_{2}^{v_{2}}\right)=E_{1} \cup E_{2}$. We next illustrate this operation.

Let $T_{1}$ and $T_{2}$ be two trees with $n$ vertices such that $T_{2}$ covers $T_{1}$ in GTS Let $E\left(P_{k}\right)$ be the edges on the path $P_{k}$ in $T_{1}$. Let $H_{1}$ and $H_{2}$ be the connected components of $T_{1}-E\left(P_{k}\right)$ containing vertices 1 and $k$ respectively. For the example of Figure 1, $H_{1}$ and $H_{2}$ are the subtrees of $T_{1}$ with vertex sets $X \cup\{1\}$ and $Y \cup\{k\}$ respectively. We also treat $H_{2}$ as a subtree of $T_{2}$ with vertex set $Y \cup\{1\}$. Therefore, $T_{1}=\left(H_{1}^{1}: P_{k}^{1}\right)^{k}: H_{2}^{k}$ and $T_{2}=\left(H_{1}^{1}: P_{k}^{1}\right)^{1}: H_{2}^{1}$. Thus, we obtain the following remark. We will use it in Section 5 .

Remark 9 Let $T_{1}$ and $T_{2}$ be two trees on $n$ vertices such that $T_{2}$ covers $T_{1}$ in $\mathrm{GTS}_{n}$. Then, $\left|P_{k}\right|+$ $\left|H_{1}\right|+\left|H_{2}\right|=n+2$. When $n$ is an even number then either all three subtrees $P_{k}, H_{1}$ and $H_{2}$ have an even number of vertices or exactly one subtree has an even number of vertices. Similarly, when $n$ is an odd number then either all three subtrees have an odd number of vertices or exactly one subtree has an odd number of vertices.

Let $T$ be a tree on $n$ vertices with $q$-Laplacian $\mathcal{L}_{T}^{q}$. We consider the characteristic polynomial $f \mathcal{L}_{T}^{q}(q, x)=\operatorname{det}\left(x I-\mathcal{L}_{T}^{q}\right)$. This is a bivariate polynomial. For a fixed vertex $v \in T$, let $\mathcal{L}_{T}^{q} \mid v$ be the submatrix obtained by deleting $v$-th row and $v$-th column from $\mathcal{L}_{T}^{q}$. Let $f^{\mathcal{L}_{T}^{q}} \mid v(q, x)=$ $\operatorname{det}\left(x I-\mathcal{L}_{T}^{q} \mid v\right)$.

The following lemma is called the general lemma which was proved by Csikvári [6] for graph polynomials in one variable $x$. We will apply it to the characteristic polynomial of $\mathcal{L}_{T}^{q}$ which is a bivariate polynomial. Since the proof is identical to that of Csikvári [6, Theorem 5.1], we omit it and merely state the result.

Lemma 10 Let $Q_{1}$ and $Q_{2}$ be two trees. For any two fixed vertices $v_{1} \in Q_{1}$ and $v_{2} \in Q_{2}$, let $T=Q_{1}^{v_{1}}: Q_{2}^{v_{2}}$. Suppose

$$
\begin{aligned}
f^{\mathcal{L}_{T}^{q}}(q, x)= & y_{1} f^{\mathcal{L}_{Q_{1}}^{q}}(q, x) f^{\mathcal{L}_{Q_{2}}^{q}}(q, x)+y_{2} f^{\mathcal{L}_{Q_{1}}^{q}}(q, x) f^{\mathcal{L}_{Q_{2}}^{q} \mid v_{2}}(q, x)+y_{2} f^{\mathcal{L}_{Q_{1}}^{q} \mid v_{1}}(q, x) f^{\mathcal{L}_{Q_{2}}^{q}}(q, x) \\
& +y_{3} f^{\mathcal{L}_{Q_{1}}^{q} \mid v_{1}}(q, x) f^{\mathcal{L}_{Q_{2}}^{q} \mid v_{2}}(q, x)
\end{aligned}
$$

where $y_{1}, y_{2}, y_{3}$ are bivariate rational functions of $q$ and $x$. Let $T_{1}$ and $T_{2}$ be two trees with $n$ vertices such that $T_{2}$ covers $T_{1}$ in $\mathrm{GTS}_{n}$ and $y_{2} f^{\mathcal{L}_{K_{2}}^{q}}(q, x)+y_{3} f^{\mathcal{L}_{K_{2}}^{q} \mid 1}(q, x) \neq 0$, where $K_{2}$ is the complete graph on 2 vertices. Then,

$$
\begin{aligned}
& f^{\mathcal{L}_{T_{1}}^{q}}(q, x)-f^{\mathcal{L}_{T_{2}}^{q}}(q, x)= y_{4}\left[y_{2} f^{\mathcal{L}_{P_{k}}^{q}}(q, x)+y_{3} f^{\mathcal{L}_{P_{k}}^{q} \mid 1}(q, x)\right]\left[y_{2} f^{\mathcal{L}_{H_{1}}^{q}}(q, x)+y_{3} f^{\mathcal{L}_{H_{1}}^{q} \mid 1}(q, x)\right] \\
& \times {\left[y_{2} f^{\mathcal{L}_{H_{2}}^{q}}(q, x)+y_{3} f_{H_{2}}^{\mathcal{L}_{1} \mid 1}(q, x)\right] } \\
& \text { where } \quad y_{4}=\frac{\left[f^{\mathcal{L}_{P_{3}}^{q} \mid 1}(q, x)-f^{\mathcal{L}_{P_{3}}^{q} \mid 2}(q, x)\right]}{\left[y_{2} f^{\mathcal{L}_{K_{2}}^{q}}(q, x)+y_{3} f^{\mathcal{L}_{K_{2}}^{q} \mid 1}(q, x)\right]^{2}}
\end{aligned}
$$


Let $Q_{1}$ and $Q_{2}$ be two trees and let $T=Q_{1}^{v_{1}}: Q_{2}^{v_{2}}$. Let $[m]=\{1,2, \ldots, m\}$ be the vertex set of $T$ and let $v_{1}=r=v_{2}$, where $\{1,2, \ldots, r\}$ and $\{r, r+1, \ldots, m\}$ be the vertex sets of $Q_{1}$ and $Q_{2}$ respectively. Let $\mathcal{L}_{Q_{1}}^{q}, \mathcal{L}_{Q_{2}}^{q}$ and $\mathcal{L}_{T}^{q}$ be the $q$-Laplacians of $Q_{1}, Q_{2}$ and $T$ respectively. We extend the notion of $\mathcal{L}_{T}^{q} \mid v$ to an arbitrary subset of $[m]$. Let $S \subseteq[m]$ and let $S^{\prime}=[m]-S$. Let $\mathcal{L}_{T}^{q} \mid S$ be the submatrix of $\mathcal{L}_{T}^{q}$ induced on the rows and columns with indices in the set $S^{\prime}$. We need the following lemma to obtain the rational functions $y_{1}, y_{2}, y_{3}$ and $y_{4}$.

Lemma 11 Let $\mathcal{L}_{Q_{1}}^{q}, \mathcal{L}_{Q_{2}}^{q}, \mathcal{L}_{T}^{q}, \mathcal{L}_{Q_{1}}^{q} \mid v_{1}$ and $\mathcal{L}_{Q_{2}}^{q} \mid v_{2}$ be the matrices as defined in the above paragraph. Let $f^{\mathcal{L}_{Q_{1}}^{q}}(q, x), f^{\mathcal{L}_{Q_{2}}^{q}}(q, x), f^{\mathcal{L}_{T}^{q}}(q, x), f^{\mathcal{L}_{Q_{1}}^{q} \mid v_{1}}(q, x)$ and $f^{\mathcal{L}_{Q_{2}}^{q} \mid v_{2}}(q, x)$ denote the characteristic polynomials of $\mathcal{L}_{Q_{1}}^{q}, \mathcal{L}_{Q_{2}}^{q}, \mathcal{L}_{T}^{q}, \mathcal{L}_{Q_{1}}^{q} \mid v_{1}$ and $\mathcal{L}_{Q_{2}}^{q} \mid v_{2}$ respectively. Then, these polynomials satisfy the condition given in (1).

Proof: Let $l_{r, r}$ denote the $(r, r)$-th entry in the bivariate polynomial matrix $x I-\mathcal{L}_{T}^{q}$. Therefore, $l_{r, r}=x-\left(1+q^{2}\left(d_{v_{1}}+d_{v_{2}}-1\right)\right)$, where $d_{v_{1}}$ and $d_{v_{2}}$ are the degrees of the vertices $v_{1}$ and $v_{2}$ in $Q_{1}$ and $Q_{2}$ respectively. Let $\mu=\left(\mu_{i}\right)_{1 \leq i \leq r-1}$ and $\nu=\left(\nu_{j}\right)_{r+1 \leq j \leq m}$ be two column vectors such that $\mu_{i}=q$ if $i$ is a neighbour of $r$ in $Q_{1}$ and $\mu_{i}=0$ otherwise. Similarly, $\nu_{j}=q$ if $j$ is a neighbour of $r$ in $Q_{2}$ and $\nu_{j}=0$ otherwise. Let $R=\{1,2, \ldots, r\}, S=\{1,2, \ldots, r-1\}, R^{\prime}=[m]-R$ and $S^{\prime}=[m]-S$. Therefore,

$$
x I-\mathcal{L}_{T}^{q}=\left[\begin{array}{ccc}
N & \mu & 0 \\
\mu^{t} & l_{r, r} & \nu^{t} \\
0 & \nu & M
\end{array}\right], \text { where } M=\left(x I-\mathcal{L}_{T}^{q} \mid R\right) \text { and } N=\left(x I-\mathcal{L}_{T}^{q} \mid S^{\prime}\right) .
$$

Clearly $\operatorname{det}(M)=f^{\mathcal{L}_{Q_{2}}^{q} \mid v_{2}}(q, x)$ and $\operatorname{det}(N)=f^{\mathcal{L}_{Q_{1}}^{q} \mid v_{1}}(q, x)$. Let $M^{\prime}=\left(x I-\mathcal{L}_{T}^{q} \mid R^{\prime}\right)$ and $N^{\prime}=\left(x I-\mathcal{L}_{T}^{q} \mid S\right)$. Then, it is simple to see the following.

$$
\begin{aligned}
\operatorname{det}\left(M^{\prime}\right) & =\operatorname{det}\left[\begin{array}{cc}
N & \mu \\
\mu^{t} & x-\left(1+q^{2}\left(d_{v_{1}}+d_{v_{2}}-1\right)\right)
\end{array}\right]=f^{\mathcal{L}_{Q_{1}}^{q}(q, x)-q^{2} d_{v_{2}} f^{\mathcal{L}_{Q_{1}}^{q} \mid v_{1}}(q, x) .} \\
\operatorname{det}\left(N^{\prime}\right) & =\operatorname{det}\left[\begin{array}{cc}
x-\left(1+q^{2}\left(d_{v_{1}}+d_{v_{2}}-1\right)\right) & \nu^{t} \\
\nu & M
\end{array}\right]=f^{\mathcal{L}_{Q_{2}}^{q}(q, x)-q^{2} d_{v_{1}} f^{\mathcal{L}_{Q_{2}}^{q} \mid v_{2}}(q, x) .}
\end{aligned}
$$

From (3), when we expand $\operatorname{det}\left(x I-\mathcal{L}_{T}^{q}\right)$ with respect to the $r$-th row, we get

$$
\begin{aligned}
& f^{\mathcal{L}_{T}^{q}}(q, x)=\operatorname{det}\left(x I-\mathcal{L}_{T}^{q}\right)=\operatorname{det}\left(M^{\prime}\right) \operatorname{det}(M)+\operatorname{det}(N) \operatorname{det}\left(N^{\prime}\right)-l_{r, r} \operatorname{det}(N) \operatorname{det}(M)
\end{aligned}
$$

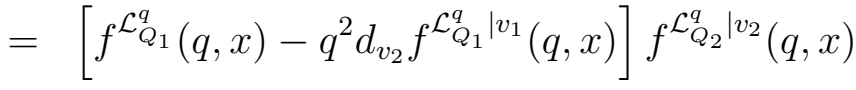

$$
\begin{aligned}
& +f^{\mathcal{L}_{Q_{1}}^{q} \mid v_{1}}(q, x)\left[f^{\mathcal{L}_{Q_{2}}^{q}}(q, x)-q^{2} d_{v_{1}} f^{\mathcal{L}_{Q_{2}}^{q} \mid v_{2}}(q, x)\right] \\
& -\left(x-\left(1+q^{2}\left(d_{v_{1}}+d_{v_{2}}-1\right)\right)\right) f^{\mathcal{L}_{Q_{1}}^{q} \mid v_{1}}(q, x) f^{\mathcal{L}_{Q_{2}}^{q} \mid v_{2}}(q, x) \\
& =f^{\mathcal{L}_{Q_{1}}^{q}}(q, x) f^{\mathcal{L}_{Q_{2}}^{q} \mid v_{2}}(q, x)+f^{\mathcal{L}_{Q_{2}}^{q}}(q, x) f^{\mathcal{L}_{Q_{1}}^{q} \mid v_{1}}(q, x) \\
& -\left(x-1+q^{2}\right) f^{\mathcal{L}_{Q_{1}}^{q} \mid v_{1}}(q, x) f^{\mathcal{L}_{Q_{2}}^{q} \mid v_{2}}(q, x),
\end{aligned}
$$

where the third equality follows from (4) and (5). The last equality follows by doing simple manipulations completing the proof. 
Let $T$ be a tree with $q$-Laplacian $\mathcal{L}_{T}^{q}$. For a fixed $q \in \mathbb{R} \backslash\{0\}$ and for a fixed vertex $v \in T$, define the auxiliary polynomial

$$
\mathcal{F}_{T}^{v}(q, x)=f^{\mathcal{L}_{T}^{q}}(q, x)-\left(x+q^{2}-1\right) f^{\mathcal{L}_{T}^{q} \mid v}(q, x) .
$$

We recall that $P_{k}, H_{1}$ and $H_{2}$ are the subtrees of $T_{1}$ and $T_{2}$, where $T_{2}$ covers $T_{1}$ in $\mathrm{GTS}_{n}$. From (1) and (6), we get the rational functions $y_{1}=0, y_{2}=1, y_{3}=-\left(x-1+q^{2}\right)$.

Theorem 12 Let $T_{1}$ and $T_{2}$ be two trees on $n$ vertices with $q$-Laplacians $\mathcal{L}_{T_{1}}^{q}$ and $\mathcal{L}_{T_{2}}^{q}$ respectively. Let $T_{2}$ cover $T_{1}$ in $\mathrm{GTS}_{n}$. Then, for all $q \in \mathbb{R} \backslash\{0\}$

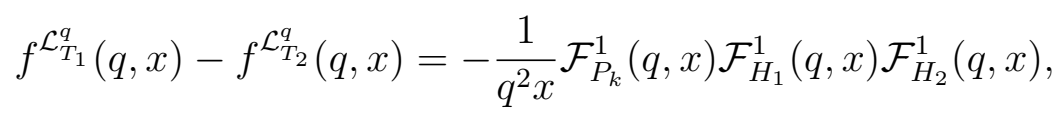

where $\mathcal{F}_{P_{k}}^{1}(q, x), \mathcal{F}_{H_{1}}^{1}(q, x)$ and $\mathcal{F}_{H_{2}}^{1}(q, x)$ are the polynomials defined in (7).

Proof: From Lemma 11 , we get $y_{1}=0, y_{2}=1, y_{3}=-\left(x-1+q^{2}\right)$. Therefore when $q \in \mathbb{R} \backslash\{0\}$

$$
y_{2} f^{\mathcal{L}_{K_{2}}^{q}}(q, x)+y_{3} f^{\mathcal{L}_{K_{2}}^{q} \mid 1}(q, x)=(x-1)^{2}-q^{2}-\left(x-1+q^{2}\right)(x-1)=-q^{2} x \neq 0 .
$$

Note that $f^{\mathcal{L}_{P_{3}}^{q} \mid 1}(q, x)-f^{\mathcal{L}_{P_{3}}^{q} \mid 2}(q, x)=-q^{2} x$. Thus, by (2), we have $y_{4}=-1 / q^{2} x$. Using Lemma 10, the proof is complete.

\section{Interlacing of eigenvalues of $\mathcal{L}_{T}^{q}$}

Let $A$ be an $n \times n$ real symmetric matrix. We order the eigenvalues of $A$ as $\lambda_{1}(A) \geq \lambda_{2}(A) \geq$ $\cdots \geq \lambda_{n}(A)$. We need the following lemma from Molitierno [11, Theorem 1.2.8 and Corollary 1.2.11].

Lemma 13 Let $A, B$ be two $n \times n$ real symmetric matrices with $B=z z^{t}$ for some column vector $z \in \mathbb{R}^{n}$. Then,

$$
\lambda_{1}(A+B) \geq \lambda_{1}(A) \geq \cdots \geq \lambda_{n}(A+B) \geq \lambda_{n}(A) .
$$

We have two interlacing lemmas about the eigenvalues of $\mathcal{L}_{T}^{q}$ when $q \in \mathbb{R}$ with either $|q| \leq 1$ or $|q|>1$. The following result is an interlacing lemma about the eigenvalues of $\mathcal{L}_{T}^{q}$ when $|q| \leq 1$.

Lemma 14 Let $T$ be a tree on $n$ vertices with a leaf vertex l. Let $T^{\prime}=T-\{l\}$. Let $\mathcal{L}_{T}^{q}$ and $\mathcal{L}_{T^{\prime}}^{q}$ be the $q$-Laplacians of $T$ and $T^{\prime}$ respectively. Then, for $q \in \mathbb{R}$ with $|q| \leq 1$,

$$
\lambda_{1}\left(\mathcal{L}_{T}^{q}\right) \geq \lambda_{1}\left(\mathcal{L}_{T^{\prime}}^{q}\right) \geq \cdots \geq \lambda_{n-1}\left(\mathcal{L}_{T^{\prime}}^{q}\right) \geq \lambda_{n}\left(\mathcal{L}_{T}^{q}\right) \geq 0
$$


Proof: We can without loss of generality assume in $T$ that $l=n$ is the deleted leaf vertex with neighbour $n-1$. Let $e_{n-1}=[0,0, \ldots, 0,1]^{t} \in \mathbb{R}^{n-1}$. Then,

$$
\begin{aligned}
\mathcal{L}_{T}^{q} & =\left[\begin{array}{cc}
q^{2} e_{n-1} e_{n-1}^{t}+\mathcal{L}_{T^{\prime}}^{q} & -q e_{n-1} \\
-q e_{n-1}^{t} & 1
\end{array}\right]=\left[\begin{array}{cc}
\mathcal{L}_{T^{\prime}}^{q} & 0 \\
0 & 0
\end{array}\right]+\left[\begin{array}{cc}
q^{2} e_{n-1} e_{n-1}^{t} & -q e_{n-1} \\
-q e_{n-1}^{t} & 1
\end{array}\right] \\
& =\left[\begin{array}{cc}
\mathcal{L}_{T^{\prime}}^{q} & 0 \\
0 & 0
\end{array}\right]+z z^{t}, \text { where } z=[0,0, \ldots, 0,-q, 1]^{t} \in \mathbb{R}^{n} .
\end{aligned}
$$

By Lemma 5, both $\mathcal{L}_{T}^{q}$ and $\mathcal{L}_{T^{\prime}}^{q}$ are positive semidefinite when $|q| \leq 1$. Therefore, all the eigenvalues of $\mathcal{L}_{T}^{q}$ and $\mathcal{L}_{T^{\prime}}^{q}$ are non-negative. Thus, by using Lemma 13 , we have $\lambda_{1}\left(\mathcal{L}_{T}^{q}\right) \geq$ $\lambda_{1}\left(\mathcal{L}_{T^{\prime}}^{q}\right) \geq \cdots \geq \lambda_{n-1}\left(\mathcal{L}_{T^{\prime}}^{q}\right) \geq \lambda_{n}\left(\mathcal{L}_{T}^{q}\right) \geq 0$ completing the proof.

From Lemma 5, $\mathcal{L}_{T}^{q}$ is not positive semidefinite when $q \in \mathbb{R}$ with $|q|>1$. We prove the following partial interlacing lemma about the eigenvalues of $\mathcal{L}_{T}^{q}$ when $|q|>1$.

Lemma 15 Let $T$ be a tree on $n$ vertices with a leaf vertex l. Let $T^{\prime}=T-\{l\}$. Let $\mathcal{L}_{T}^{q}$ and $\mathcal{L}_{T^{\prime}}^{q}$ be the $q$-Laplacians of $T$ and $T^{\prime}$ respectively. Then, for $q \in \mathbb{R}$ with $|q|>1$,

$$
\lambda_{1}\left(\mathcal{L}_{T}^{q}\right) \geq \lambda_{1}\left(\mathcal{L}_{T^{\prime}}^{q}\right) \geq \cdots \geq \lambda_{n-2}\left(\mathcal{L}_{T^{\prime}}^{q}\right) \geq \lambda_{n-1}\left(\mathcal{L}_{T}^{q}\right)>0>\lambda_{n}\left(\mathcal{L}_{T}^{q}\right) \geq \lambda_{n-1}\left(\mathcal{L}_{T^{\prime}}^{q}\right) .
$$

Proof: As done in Lemma 14, we assume the vertex $l=n$ and that its neighbour is vertex $n-1$. Thus, we obtain $\mathcal{L}_{T}^{q}=A+B$, where

$$
A=\left[\begin{array}{cc}
\mathcal{L}_{T^{\prime}}^{q} & 0 \\
0 & 0
\end{array}\right] \text { and } B=z z^{t} \text { with } z=[0,0, \ldots, 0,-q, 1]^{t} \in \mathbb{R}^{n}
$$

By Lemma 5 , both $\mathcal{L}_{T}^{q}$ and $\mathcal{L}_{T^{\prime}}^{q}$ have exactly one negative eigenvalue and both $\mathcal{L}_{T}^{q}$ and $\mathcal{L}_{T^{\prime}}^{q}$ are invertible when $|q|>1$. Therefore, the eigenvalues of $A$ are the following: $\lambda_{1}\left(\mathcal{L}_{T^{\prime}}^{q}\right) \geq \cdots \geq$ $\lambda_{n-2}\left(\mathcal{L}_{T^{\prime}}^{q}\right)>0>\lambda_{n-1}\left(\mathcal{L}_{T^{\prime}}^{q}\right)$. Thus, by Lemma $13, \lambda_{1}\left(\mathcal{L}_{T}^{q}\right) \geq \lambda_{1}\left(\mathcal{L}_{T^{\prime}}^{q}\right) \geq \cdots \geq \lambda_{n-1}\left(\mathcal{L}_{T}^{q}\right)>0>$ $\lambda_{n}\left(\mathcal{L}_{T}^{q}\right) \geq \lambda_{n-1}\left(\mathcal{L}_{T^{\prime}}^{q}\right)$ completing the proof.

Let $T$ be a tree on $n$ vertices with $q$-Laplacian $\mathcal{L}_{T}^{q}$. Let $\lambda_{\max }\left(\mathcal{L}_{T}^{q}\right), \lambda_{\min }\left(\mathcal{L}_{T}^{q}\right)$ and $\lambda_{a}\left(\mathcal{L}_{T}^{q}\right)=$ $\lambda_{n-1}\left(\mathcal{L}_{T}^{q}\right)$ denote the largest, the smallest and the second smallest eigenvalues of $\mathcal{L}_{T}^{q}$ respectively. We need the following corollaries of Lemmas 14 and 15 .

Corollary 16 Let $T$ be a tree with $n$ vertices and let $T^{\prime}$ be a subtree of $T$. Let $\mathcal{L}_{T}^{q}$ and $\mathcal{L}_{T^{\prime}}^{q}$ be the $q$-Laplacians of $T$ and $T^{\prime}$ respectively. Then, for all $q \in \mathbb{R}, \lambda_{\max }\left(\mathcal{L}_{T^{\prime}}^{q}\right) \leq \lambda_{\max }\left(\mathcal{L}_{T}^{q}\right)$.

Proof: Let $T^{\prime}=T_{0}$ be the given subtree of $T$ and let $m$ be the number of vertices in $T_{0}$. Construct a new tree $T_{1}$ by adding a leaf vertex in $T_{0}$ such that $T_{1}$ is again a subtree of $T$. Thus, from Lemmas 14 and 15, $\lambda_{\max }\left(\mathcal{L}_{T_{0}}^{q}\right) \leq \lambda_{\max }\left(\mathcal{L}_{T_{1}}^{q}\right)$ for all $q \in \mathbb{R}$. By repeating this process we get a sequence of subtrees $T^{\prime}=T_{0}, T_{1}, \ldots, T_{n-m}=T$ of $T$ with $\lambda_{\max }\left(\mathcal{L}_{T^{\prime}}^{q}\right)=\lambda_{\max }\left(\mathcal{L}_{T_{0}}^{q}\right) \leq \lambda_{\max }\left(\mathcal{L}_{T_{1}}^{q}\right) \leq \cdots \leq$ $\lambda_{\max }\left(\mathcal{L}_{T_{n-m}}^{q}\right)=\lambda_{\max }\left(\mathcal{L}_{T}^{q}\right)$ and hence, completing the proof.

By using similar argument as done in the proof of Corollary 16 , we see that $\lambda_{a}\left(\mathcal{L}_{T^{\prime}}^{q}\right) \geq \lambda_{a}\left(\mathcal{L}_{T}^{q}\right)$, where $T^{\prime}$ is a subtree of $T$. Thus, we obtain the following corollary of Lemmas 14 and 15 .

Corollary 17 Using the notations of Theorem 12 let $P_{k}, H_{1}$ and $H_{2}$ be the subtrees of both $T_{1}$ and $T_{2}$. Then, for all $q \in \mathbb{R}$, we have $\max \left(\lambda_{a}\left(\mathcal{L}_{T_{1}}^{q}\right), \lambda_{a}\left(\mathcal{L}_{T_{2}}^{q}\right)\right) \leq \min \left(\lambda_{a}\left(\mathcal{L}_{P_{k}}^{q}\right), \lambda_{a}\left(\mathcal{L}_{H_{1}}^{q}\right), \lambda_{a}\left(\mathcal{L}_{H_{2}}^{q}\right)\right)$. 


\section{$5 \quad$ Auxiliary polynomial $\mathcal{F}_{T}^{v}(q, x)$}

Let $T$ be a tree on $n$ vertices with $q$-Laplacian $\mathcal{L}_{T}^{q}$. We recall the definition of the polynomial $\mathcal{F}_{T}^{v}(q, x)$ defined in (7) as follows:

$$
\mathcal{F}_{T}^{v}(q, x)=f^{\mathcal{L}_{T}^{q}}(q, x)-\left(x-1+q^{2}\right) f^{\mathcal{L}_{T}^{q} \mid v}(q, x)=f^{\mathcal{L}_{T}^{q}}(q, x)-\left(x-\operatorname{det}\left(\mathcal{L}_{T}^{q}\right)\right) f^{\mathcal{L}_{T}^{q} \mid v}(q, x) .
$$

We think of $\mathcal{F}_{T}^{v}(q, x)$ as a univariate polynomial once a real value for $q$ is assigned. From Theorem 12 , we recall that when a tree $T_{2}$ covers an another tree $T_{1}$ in $\mathrm{GTS}_{n}, f^{\mathcal{L}_{T_{1}}^{q}}(q, x)-f \mathcal{L}_{T_{2}}^{q}(q, x)$ is a product of three auxiliary polynomials of subtrees $P_{k}, H_{1}$, and $H_{2}$ of $T_{1}$ and $T_{2}$. Therefore the roots of this difference polynomial is the multiset union of the roots of auxiliary polynomials of these subtrees $P_{k}, H_{1}$, and $H_{2}$. Thus, to prove Theorem 2, we need to determine the location of all these roots and decide the sign of $f^{\mathcal{L}_{T_{1}}^{q}}(q, x)-f^{\mathcal{L}_{T_{2}}^{4}}(q, x)$ for a fixed $q \in$ $\mathbb{R} \backslash\{0\}$ and when $x \in\left[\left(-\infty, t_{1}\right) \cup\left(t_{2}, \infty\right)\right]-\{0\}$, where $t_{1}=\max \left(\lambda_{a}\left(\mathcal{L}_{T_{1}}^{q}\right), \lambda_{a}\left(\mathcal{L}_{T_{2}}^{q}\right)\right)$ and $t_{2}=\min \left(\lambda_{\max }\left(\mathcal{L}_{T_{1}}^{q}\right), \lambda_{\max }\left(\mathcal{L}_{T_{2}}^{q}\right)\right)$.

Lemma 18 For a tree $T$ on $n>1$ vertices with $q$-Laplacian $\mathcal{L}_{T}^{q}$, the degree of $\mathcal{F}_{T}^{v}(q, x)$ is $n-1$ and the coefficient of $x^{n-1}$ in $\mathcal{F}_{T}^{v}(q, x)$ is $-q^{2} d_{v}$. Further, for all $q \in \mathbb{R} \backslash\{0\}$, zero is a root of $\mathcal{F}_{T}^{v}(q, x)$.

Proof: Without loss of generality we assume that the first row of $\mathcal{L}_{T}^{q}$ is indexed by the vertex $v$. Let $\alpha=\left(\alpha_{i}\right)_{2 \leq i \leq n}$ be a column vector such that $\alpha_{i}=q$ if $v$ is adjacent to the vertex $i$ and $\alpha_{i}=0$ otherwise. Therefore

$$
\begin{aligned}
f^{\mathcal{L}_{T}^{q}}(q, x) & =\operatorname{det}\left[\begin{array}{cc}
x-1-q^{2}\left(d_{v}-1\right) & \alpha^{t} \\
\alpha & x I-\mathcal{L}_{T}^{q} \mid v
\end{array}\right] \\
& =\operatorname{det}\left[\begin{array}{cc}
x-1+q^{2} & \alpha^{t} \\
0 & x I-\mathcal{L}_{T}^{q} \mid v
\end{array}\right]+\operatorname{det}\left[\begin{array}{cc}
-q^{2} d_{v} & \alpha^{t} \\
\alpha & x I-\mathcal{L}_{T}^{q} \mid v
\end{array}\right] \\
& =\left(x-1+q^{2}\right) f^{\mathcal{L}_{T}^{q} \mid v}(q, x)+\operatorname{det}\left[\begin{array}{cc}
-q^{2} d_{v} & \alpha^{t} \\
\alpha & x I-\mathcal{L}_{T}^{q} \mid v
\end{array}\right]
\end{aligned}
$$

From the definition of $\mathcal{F}_{T}^{v}(q, x)$ and by using $(8)$, it is easy to see that the degree of the polynomial $\mathcal{F}_{T}^{v}(q, x)$ in the variable $x$ is $n-1$ and the coefficient of $x^{n-1}$ in $\mathcal{F}_{T}^{v}(q, x)$ is $-q^{2} d_{v}$.

Nagar and Sivasubramanian [12, Remark 13] proved that $f^{\mathcal{L}_{T}^{q}} \mid v(q, 0)=(-1)^{n-1}$. Thus, when $x=0$ from Lemma 5 , we get the following.

$$
\mathcal{F}_{T}^{v}(q, 0)=f^{\mathcal{L}_{T}^{q}}(q, 0)-\left(q^{2}-1\right) f^{\mathcal{L}_{T}^{q} \mid v}(q, 0)=(-1)^{n} \operatorname{det}\left(\mathcal{L}_{T}^{q}\right)-\left(q^{2}-1\right)(-1)^{n-1}=0 .
$$

Thus, zero is a root of $\mathcal{F}_{T}^{v}(q, x)$ and hence the proof is complete.

For a fixed $q \in \mathbb{R} \backslash\{0\}$, from Lemma 18 , it is easy to see that for large positive $x$ the function $\mathcal{F}_{T}^{v}(q, x)$ is negative. When $|q| \leq 1$, we next prove that the multiplicity of zero as a root of $\mathcal{F}_{T}^{v}(q, x)$ is one and determine the location of its $n-2$ non-zero roots. Let the eigenvalues of $\mathcal{L}_{T}^{q} \mid v$ be $\lambda_{1}\left(\mathcal{L}_{T}^{q} \mid v\right) \geq \lambda_{2}\left(\mathcal{L}_{T}^{q} \mid v\right) \geq \cdots \geq \lambda_{n-1}\left(\mathcal{L}_{T}^{q} \mid v\right)$. Let $\sigma\left(\mathcal{L}_{T}^{q} \mid v\right)=\left\{\lambda_{1}\left(\mathcal{L}_{T}^{q} \mid v\right), \ldots, \lambda_{n-1}\left(\mathcal{L}_{T}^{q} \mid v\right)\right\}$ be the multiset of eigenvalues of $\mathcal{L}_{T}^{q} \mid v$. From [12, Corollary 12], it is simple to see that all the 
eigenvalues of $\mathcal{L}_{T}^{q} \mid v$ are non-negative. Let $\sigma\left(\mathcal{L}_{T}^{q}\right)=\left\{\lambda_{1}\left(\mathcal{L}_{T}^{q}\right), \ldots, \lambda_{n-1}\left(\mathcal{L}_{T}^{q}\right)\right\}$ be the multisets of the $n-1$ largest eigenvalues of $\mathcal{L}_{T}^{q}$. Motivated by Csikvári [6, the third part of Theorem 7.3] when $q \in \mathbb{R}$ with $|q| \leq 1$, we obtain the following result.

Lemma 19 Let $T$ be a tree on $n>1$ vertices and let $v \in T$. Then, for a fixed $q \in \mathbb{R} \backslash\{0\}$ with $|q| \leq 1$, each non-zero root of $\mathcal{F}_{T}^{v}(q, x)$ lies in the interval $\left[\lambda_{a}\left(\mathcal{L}_{T}^{q}\right), \lambda_{\max }\left(\mathcal{L}_{T}^{q}\right)\right]$.

Proof: Using the Interlacing Theorem for eigenvalues of symmetric matrices (see Godsil and Royle [8, page 193]), we get the following.

$$
\lambda_{1}\left(\mathcal{L}_{T}^{q}\right) \geq \lambda_{1}\left(\mathcal{L}_{T}^{q} \mid v\right) \geq \cdots \geq \lambda_{i}\left(\mathcal{L}_{T}^{q}\right) \geq \lambda_{i}\left(\mathcal{L}_{T}^{q} \mid v\right) \geq \cdots \geq \lambda_{n-1}\left(\mathcal{L}_{T}^{q} \mid v\right) \geq \lambda_{n}\left(\mathcal{L}_{T}^{q}\right) .
$$

We break the proof into two cases when $1-q^{2} \geq \lambda_{n-1}\left(\mathcal{L}_{T}^{q}\right)$ and when $1-q^{2}<\lambda_{n-1}\left(\mathcal{L}_{T}^{q}\right)$. For both the cases, the proof is identical. Thus, we only consider the case when $1-q^{2} \geq \lambda_{n-1}\left(\mathcal{L}_{T}^{q}\right)$.

Firstly we assume that $\sigma\left(\mathcal{L}_{T}^{q}\right) \cap\left[\sigma\left(\mathcal{L}_{T}^{q} \mid v\right) \cup\left\{1-q^{2}\right\}\right]=\emptyset$, where $\cap$ and $\mathbb{U}$ denote the multiset intersection and multiset union respectively. Therefore from (9), the multiplicity of each eigenvalue of $\mathcal{L}_{T}^{q}$ and $\mathcal{L}_{T}^{q} \mid v$ is one and we get

$$
\lambda_{1}\left(\mathcal{L}_{T}^{q}\right)>\lambda_{1}\left(\mathcal{L}_{T}^{q} \mid v\right)>\cdots>\lambda_{n-1}\left(\mathcal{L}_{T}^{q}\right)>\lambda_{n-1}\left(\mathcal{L}_{T}^{q} \mid v\right) \text { with } 1-q^{2}>\lambda_{n-1}\left(\mathcal{L}_{T}^{q}\right) .
$$

It is easy to see that

$$
\begin{aligned}
\lambda_{\max }\left(\mathcal{L}_{T}^{q}\right) & \geq \frac{\sum_{i=0}^{n} \lambda_{i}\left(\mathcal{L}_{T}^{q}\right)}{n}=\frac{\operatorname{Trace}\left(\mathcal{L}_{T}^{q}\right)}{n}=\frac{\sum_{v \in T} 1+q^{2}\left(d_{v}-1\right)}{n}=\frac{n+q^{2}(n-2)}{n} \\
& \geq 1-q^{2} .
\end{aligned}
$$

Thus, from (10) and (11), $1-q^{2}$ is sandwiched between two eigenvalues of $\mathcal{L}_{T}^{q}$. Thus, for some $i$ with $1 \leq i \leq n-2$, we get

$$
\lambda_{i}\left(\mathcal{L}_{T}^{q}\right)>\lambda_{i}\left(\mathcal{L}_{T}^{q} \mid v\right)>\lambda_{i+1}\left(\mathcal{L}_{T}^{q}\right) \text { with } \lambda_{i}\left(\mathcal{L}_{T}^{q}\right)>m_{1} \geq m_{2}>\lambda_{i+1}\left(\mathcal{L}_{T}^{q}\right),
$$

where $m_{1}=\max \left(1-q^{2}, \lambda_{i}\left(\mathcal{L}_{T}^{q} \mid v\right)\right)$ and $m_{2}=\min \left(1-q^{2}, \lambda_{i}\left(\mathcal{L}_{T}^{q} \mid v\right)\right)$. Recall for a fixed $q \in$ $\mathbb{R} \backslash\{0\}$, the polynomial $\mathcal{F}_{T}^{v}(q, x)$ is univariate in the variable $x$. Thus, by the intermediate value theorem (IVT henceforth), as both the quantities $\mathcal{F}_{T}^{v}\left(q, m_{1}\right)=f^{\mathcal{L}}\left(q, m_{1}\right)$ and $\mathcal{F}_{T}^{v}\left(q, m_{2}\right)=$ $f^{\mathcal{L}_{T}^{q}}\left(q, m_{2}\right)$ have same sign, either both are positive or both are negative.

From Lemma 18, we recall that for large positive $x \in \mathbb{R}, \mathcal{F}_{T}^{v}(q, x)$ is negative. When $1<$ $i, \lambda_{1}\left(\mathcal{L}_{T}^{q}\right)>\lambda_{1}\left(\mathcal{L}_{T}^{q} \mid v\right)>m_{1}$ and the coefficient of $x^{n-1}$ in $f^{\mathcal{L}_{T}^{q}} \mid v(q, x)$ is positive. Therefore $\mathcal{F}_{T}^{v}\left(q, \lambda_{1}\left(\mathcal{L}_{T}^{q}\right)\right)=-\left(\lambda_{1}\left(\mathcal{L}_{T}^{q}\right)-1+q^{2}\right) f^{\mathcal{L}_{T}^{q}} \mid v\left(q, \lambda_{1}\left(\mathcal{L}_{T}^{q}\right)\right)$ is negative. Similarly when $2<i$, the coefficient of $x^{n}$ in $f_{\mathcal{L}_{T}^{q}}^{q}(q, x)$ is positive and $\lambda_{1}\left(\mathcal{L}_{T}^{q}\right)>\lambda_{1}\left(\mathcal{L}_{T}^{q} \mid v\right)>\lambda_{2}\left(\mathcal{L}_{T}^{q}\right)>m_{1}$. Thus, by the IVT, $\mathcal{F}_{T}^{v}\left(q, \lambda_{1}\left(\mathcal{L}_{T}^{q} \mid v\right)\right)=f^{\mathcal{L}_{T}^{q}}\left(q, \lambda_{1}\left(\mathcal{L}_{T}^{q} \mid v\right)\right)$ is again negative. By using identical arguments when $x \in\left\{\lambda_{2}\left(\mathcal{L}_{T}^{q}\right), \lambda_{2}\left(\mathcal{L}_{T}^{q} \mid v\right)\right\}$ with $\lambda_{2}\left(\mathcal{L}_{T}^{q} \mid v\right)>m_{1}, \mathcal{F}_{T}^{v}(q, x)$ is positive. $\mathcal{F}_{T}^{v}(q, x)$ is negative if $j$ is odd and positive if $j$ is even. But when $j=i$, both the quantities $\mathcal{F}_{T}^{v}\left(q, m_{1}\right)$ and $\mathcal{F}_{T}^{v}\left(q, m_{2}\right)$ have the same sign. Therefore, it is easy to extend this for all $j$ with $1 \leq j \leq n-1$. Thus, when $x \in\left\{\lambda_{j}\left(\mathcal{L}_{T}^{q}\right), \lambda_{j}\left(\mathcal{L}_{T}^{q} \mid v\right)\right\} \mathcal{F}_{T}^{v}(q, x)$ is negative if $j$ is odd and positive if $j$ is even. Hence, there must be a root of $\mathcal{F}_{T}^{v}(q, x)$ in the interval $\left[\lambda_{j+1}\left(\mathcal{L}_{T}^{q}\right), \lambda_{j}\left(\mathcal{L}_{T}^{q} \mid v\right)\right]$ for $j=1,2, \ldots, n-2$. See Example 21 for better interpretation. 
Secondly we assume that $\sigma\left(\mathcal{L}_{T}^{q}\right) \cap\left[\sigma\left(\mathcal{L}_{T}^{q} \mid v\right) \cup\left\{1-q^{2}\right\}\right]=\left\{\lambda_{1}, \lambda_{2}, \ldots, \lambda_{c}\right\}$. Clearly $\lambda_{1}, \lambda_{2}$, $\ldots, \lambda_{c}$ are the roots of $\mathcal{F}_{T}^{v}(q, x)$ and these lie in the interval $\left[\lambda_{a}\left(\mathcal{L}_{T}^{q}\right), \lambda_{\max }\left(\mathcal{L}_{T}^{q}\right)\right]$. From (9), it is clear that the multiplicity of each eigenvalue not containing in $\left\{\lambda_{1}, \ldots, \lambda_{c}\right\}$ of $\mathcal{L}_{T}^{q}$ and $\mathcal{L}_{T}^{q} \mid v$ is one. Therefore the remaining eigenvalues of $\mathcal{L}_{T}^{q}$ and $\mathcal{L}_{T}^{q} \mid v$ satisfy an identical relation as given in (10). Thus, by using similar arguments as done in the above paragraph, it is easy to determine the location of the remaining $n-c-2$ roots of $\mathcal{F}_{T}^{v}(q, x)$ by locating the roots of the following polynomial.

$$
\frac{\mathcal{F}_{T}^{v}(q, x)}{\prod_{i=1}^{c}\left(x-\lambda_{i}\right)}=\frac{f^{\mathcal{L}_{T}^{q}}(q, x)}{\prod_{i=1}^{c}\left(x-\lambda_{i}\right)}-\frac{\left(x-1+q^{2}\right) f^{\mathcal{L}_{T}^{q} \mid v}(q, x)}{\prod_{i=1}^{c}\left(x-\lambda_{i}\right)} .
$$

Thus, for $q \in \mathbb{R} \backslash\{0\}$ with $|q| \leq 1$, all the non-zero roots of $\mathcal{F}_{T}^{v}(q, x)$ lie in the interval $\left[\lambda_{a}\left(\mathcal{L}_{T}^{q}\right), \lambda_{\max }\left(\mathcal{L}_{T}^{q}\right)\right]$. The proof is complete.

When $q \in \mathbb{R}$ with $|q|>1$, the proof of the following lemma is identical to the proof of Lemma 19.

Lemma 20 Let $T$ be a tree on $n>1$ vertices and let $v \in T$. Then, for all $q \in \mathbb{R}$ with $|q|>1$, each non-zero roots of $\mathcal{F}_{T}^{v}(q, x)$ lies in the interval $\left[\lambda_{a}\left(\mathcal{L}_{T}^{q}\right), \lambda_{\max }\left(\mathcal{L}_{T}^{q}\right)\right]$.

Proof: For a fixed $q \in \mathbb{R}$ with $|q|>1$ from Lemma $5, \lambda_{n}\left(\mathcal{L}_{T}^{q}\right)<0, \lambda_{n-1}\left(\mathcal{L}_{T}^{q}\right)>0$ and $\operatorname{det}\left(\mathcal{L}_{T}^{q}\right)=1-q^{2}<0$. We recall that $\lambda_{n-1}\left(\mathcal{L}_{T}^{q} \mid v\right) \geq 0$. Therefore, the interlacing theorem for eigenvalues of symmetric matrices gives the following.

$$
\lambda_{1}\left(\mathcal{L}_{T}^{q}\right) \geq \lambda_{1}\left(\mathcal{L}_{T}^{q} \mid v\right) \geq \cdots \geq \lambda_{n-1}\left(\mathcal{L}_{T}^{q} \mid v\right)>\max \left(1-q^{2}, \lambda_{n}\left(\mathcal{L}_{T}^{q}\right)\right) \text { geq } \min \left(1-q^{2}, \lambda_{n}\left(\mathcal{L}_{T}^{q}\right)\right) .
$$

By using identical arguments as done in the proof of Lemma 19, all the non-zero roots of $\mathcal{F}_{T}^{v}(q, x)$ lie in the interval $\left[\lambda_{a}\left(\mathcal{L}_{T}^{q}\right), \lambda_{\max }\left(\mathcal{L}_{T}^{q} \mid v\right)\right]$ for all $q \in \mathbb{R}$ with $|q|>1$. The proof is complete.

The following example illustrates Lemmas 19 and 20.

Example 21 Let $T_{1}$ be the tree given in Figure 4 . For all $x \in\left[\lambda_{\min }\left(\mathcal{L}_{T_{1}}^{q}\right), \lambda_{\max }\left(\mathcal{L}_{T_{1}}^{q}\right)\right]$ with $q=0.5$ and $q=1.5$ some values of $f^{\mathcal{L}_{T_{1}}^{q}}(q, x), f^{\mathcal{L}_{T_{1}}^{q} \mid 1}(q, x)$ and $\mathcal{F}_{T_{1}}^{1}(q, x)$ are drawn in Figures 2 and 3 respectively. 1 is an eigenvalue of both the matrices $\mathcal{L}_{T_{1}}^{q}$ and $\mathcal{L}_{T_{1}}^{q} \mid 1$. Thus, 1 is a root of $\mathcal{F}_{T_{1}}^{\mathrm{1}}(q, x)$. Here, the solid red, dotted blue and thick solid black lines are used for $f^{\mathcal{L}_{T_{1}}^{q}}(q, x), f^{\mathcal{L}_{T_{1}}^{q} \mid 1}(q, x)$ and $\mathcal{F}_{T}^{1}(q, x)$ respectively. Arrows on lines are used for the behaviour of these polynomials when $x$ decreases from $\lambda_{\max }\left(\mathcal{L}_{T_{1}}^{q}\right)$ to $\lambda_{\min }\left(\mathcal{L}_{T_{1}}^{q}\right)$. These polynomials were drawn by using the computer package SageMath.

We recall that zero is a root of $\mathcal{F}_{T}^{v}(q, x)$ with multiplicity one and the second smallest root of $\mathcal{F}_{T}^{v}(q, x)$ lies in the interval $\left[\lambda_{a}\left(\mathcal{L}_{T}^{q}\right), \lambda_{n-2}\left(\mathcal{L}_{T}^{q} \mid v\right)\right]$, where $\lambda_{a}\left(\mathcal{L}_{T}^{q}\right)>0$ and $\lambda_{n-2}\left(\mathcal{L}_{T}^{q} \mid v\right)>0$ are the second smallest eigenvalues of $\mathcal{L}_{T}^{q}$ and $\mathcal{L}_{T}^{q} \mid v$ respectively. Thus, by Lemmas 18, 19 and 20, we obtain the following.

Remark 22 Let $T$ be a tree on $n$ vertices and let $v \in T$. Then, for a fixed $q \in \mathbb{R} \backslash\{0\}$ and for $x \in(-\infty, 0)$ the polynomial $\mathcal{F}_{T}^{v}(q, x)$ is positive when $n$ is even and negative when $n$ is odd. Moreover, by the IVT, for all $x \in\left(0, \lambda_{a}\left(\mathcal{L}_{T}^{q}\right)\right)$, we have $\mathcal{F}_{T}^{v}(q, x)<0$ when $n$ is even and $\mathcal{F}_{T}^{v}(q, x)>0$ when $n$ is odd. 


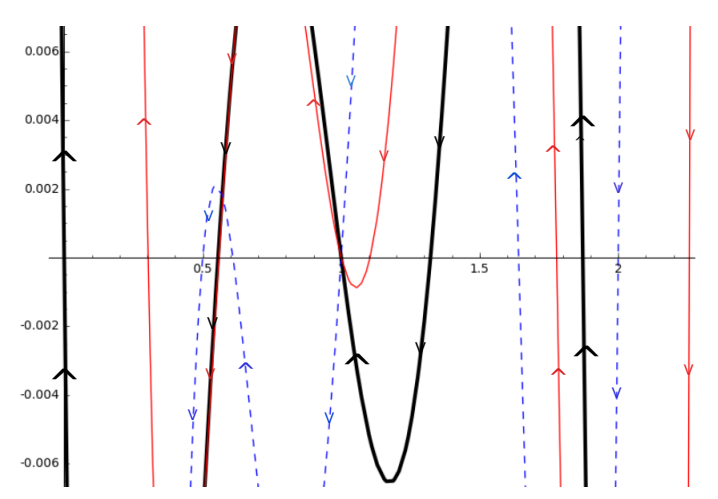

Figure 2: The values of $f^{\mathcal{L}_{T_{1}}^{q}}(0.5, x)$, $f^{\mathcal{L}_{T_{1}}^{q} \mid 1}(0.5, x)$ and $\mathcal{F}_{T_{1}}^{1}(0.5, x)$.

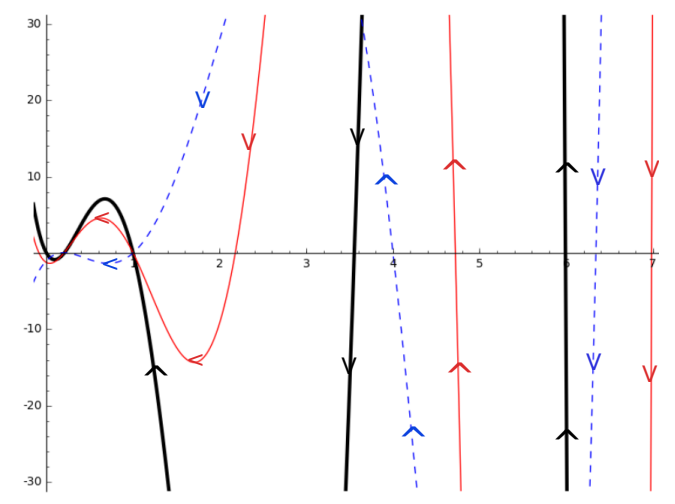

Figure 3: The values of $f^{\mathcal{L}_{T_{1}}^{q}}(1.5, x)$, $f^{\mathcal{L}_{T_{1}}^{q} \mid 1}(1.5, x)$ and $\mathcal{F}_{T_{1}}^{1}(1.5, x)$.

Let $T_{1}$ and $T_{2}$ be two trees with $n$ vertices such that $T_{2}$ covers $T_{1}$ in $\mathrm{GTS}_{n}$. For convenience, from Theorem 12 , we define

$$
\mathcal{D}_{T_{2}}^{T_{1}}(q, x)=f^{\mathcal{L}_{T_{1}}^{q}}(q, x)-f^{\mathcal{L}_{T_{2}}^{q}}(q, x)=-\frac{1}{q^{2} x} \mathcal{F}_{P_{k}}^{1}(q, x) \mathcal{F}_{H_{1}}^{1}(q, x) \mathcal{F}_{H_{2}}^{1}(q, x), \text { where } q \neq 0 .
$$

From Lemma 18 , zero is a root of all the three polynomials $\mathcal{F}_{P_{k}}^{1}(q, x), \mathcal{F}_{H_{1}}^{1}(q, x)$ and $\mathcal{F}_{H_{2}}^{1}(q, x)$ with multiplicity one. Thus, from (12), zero is a root of $\mathcal{D}_{T_{2}}^{T_{1}}(q, x)$ with multiplicity two. We need the following lemma in Section 6 .

Lemma 23 Let $T_{1}$ and $T_{2}$ be two trees with $n$ vertices such that $T_{2}$ covers $T_{1}$ in $\mathrm{GTS}_{n}$. Let $\mathcal{D}_{T_{2}}^{T_{1}}(q, x)$ be the polynomial defined in $(12)$. Then, for $i=1,2$ and for a fixed $q \in \mathbb{R} \backslash\{0\}$ when $x \in\left(-\infty, \lambda_{a}\left(\mathcal{L}_{T_{i}}^{q}\right)\right)-\{0\}$, we have $\mathcal{D}_{T_{2}}^{T_{1}}(q, x)>0$ if $n$ is even and $\mathcal{D}_{T_{2}}^{T_{1}}(q, x)<0$ if $n$ is odd.

Proof: By Corollary 17 and Remark 22, for a fixed $q \in \mathbb{R} \backslash\{0\}$ and when $x \in\left(0, \lambda_{a}\left(\mathcal{L}_{T_{i}}^{q}\right)\right)$, each polynomial from $\mathcal{F}_{P_{k}}^{1}(q, x), \mathcal{F}_{H_{1}}^{1}(q, x)$, and $\mathcal{F}_{H_{2}}^{1}(q, x)$ evaluates to a negative quantity if the number of vertices of $P_{k}, H_{1}$ and $H_{2}$ are even respectively. Similarly, each polynomial from $\mathcal{F}_{P_{k}}^{1}(q, x), \mathcal{F}_{H_{1}}^{1}(q, x)$, and $\mathcal{F}_{H_{2}}^{1}(q, x)$ evaluates to a positive quantity if the number of vertices of $P_{k}, H_{1}$ and $H_{2}$ are odd respectively.

When $n$ is an even number, then from Remark 9 , for all $x \in\left(0, \lambda_{a}\left(\mathcal{L}_{T_{i}}^{q}\right)\right)$, either all the three polynomials $\mathcal{F}_{P_{k}}^{1}(q, x), \mathcal{F}_{H_{1}}^{1}(q, x)$, and $\mathcal{F}_{H_{2}}^{1}(q, x)$ evaluate to negative quantities or exactly one polynomial evaluates to a negative quantity and other two evaluate to positive quantities. Thus, by using (12), we get $\mathcal{D}_{T_{2}}^{T_{1}}(q, x)>0$ for all $x \in\left(0, \lambda_{a}\left(\mathcal{L}_{T_{i}}^{q}\right)\right)$. Similarly, when $n$ is an odd number, then by Remark 9 and (12), $\mathcal{D}_{T_{2}}^{T_{1}}(q, x)<0$ for all $x \in\left(0, \lambda_{a}\left(\mathcal{L}_{T_{i}}^{q}\right)\right)$. By similar arguments, it is simple to see that for all $x \in(-\infty, 0), \mathcal{D}_{T_{2}}^{T_{1}}(q, x)>0$ when $n$ is even and $\mathcal{D}_{T_{2}}^{T_{1}}(q, x)<0$ when $n$ is odd. The proof is complete.

The following lemma is an easy consequence of Lemmas 19 and 20 and Corollary 16.

Lemma 24 Let $T_{1}$ and $T_{2}$ be two trees with $n$ vertices such that $T_{2}$ covers $T_{1}$ in $\mathrm{GTS}_{n}$. Then for a fixed $q \in \mathbb{R} \backslash\{0\}, \mathcal{D}_{T_{2}}^{T_{1}}(q, x)>0$ for all $x>\lambda_{\max }\left(\mathcal{L}_{T_{i}}^{q}\right)>0$, where $i=1,2$. 
Proof: We recall that $P_{k}, H_{1}$ and $H_{2}$ are the subtrees of $T_{1}$ and $T_{2}$. From Corollary 16, we see that $\max \left(\lambda_{\max }\left(\mathcal{L}_{P_{k}}^{q}\right), \lambda_{\max }\left(\mathcal{L}_{H_{1}}^{q}\right), \lambda_{\max }\left(\mathcal{L}_{H_{2}}^{q}\right)\right) \leq \min \left(\lambda_{\max }\left(\mathcal{L}_{T_{1}}^{q}\right), \lambda_{\max }\left(\mathcal{L}_{T_{2}}^{q}\right)\right)$. Thus, from Lemmas 19 and 20, the polynomials $\mathcal{F}_{P_{k}}^{1}(q, x), \mathcal{F}_{H_{1}}^{1}(q, x)$ and $\mathcal{F}_{H_{2}}^{1}(q, x)$ are negative for all $x>$ $\lambda_{\max }\left(\mathcal{L}_{T_{i}}^{q}\right)>0$ for $i=1,2$. Thus, using (12), completes the proof.

\section{Proof of Theorem 2}

We give the proof of Theorem 2 in three subsections one for each eigenvalue. It is sufficient to prove the result for each pair of covering trees on $\mathrm{GTS}_{n}$. The following remark is straight forward from the definition of $f^{\mathcal{L}_{T}^{q}}(q, x)$.

Remark 25 For a fixed $q \in \mathbb{R}$ and for $x \in\left(-\infty, \lambda_{\min }\left(\mathcal{L}_{T}^{q}\right)\right)$, the polynomial $f \mathcal{L}_{T}^{q}(q, x)$ evaluates to a positive quantity when $n$ is even and negative quantity when $n$ is odd. Moreover, when $x \in$ $\left[\lambda_{\min }\left(\mathcal{L}_{T}^{q}\right), \lambda_{a}\left(\mathcal{L}_{T}^{q}\right)\right]$, we have $f^{\mathcal{L}_{T}^{q}}(q, x) \leq 0$ if $n$ is even and $f^{q} \mathcal{L}_{T}(q, x) \geq 0$ if $n$ is odd. We also see that $f^{\mathcal{L}_{T}^{q}}(q, x)>0$ for all $x>\lambda_{\max }\left(\mathcal{L}_{T}^{q}\right)$.

\section{1 $\lambda_{\max }\left(\mathcal{L}_{T}^{q}\right)$}

The following result is about monotonicity of the largest eigenvalue of $\mathcal{L}_{T}^{q}$ of a tree $T$ on $\mathrm{GTS}_{n}$.

Theorem 26 Let $T_{1}$ and $T_{2}$ be two trees with $n$ vertices such that $T_{2}$ covers $T_{1}$ in $\mathrm{GTS}_{n}$. Then, for all $q \in \mathbb{R} \backslash\{0\}$, we have $\lambda_{\max }\left(\mathcal{L}_{T_{1}}^{q}\right) \leq \lambda_{\max }\left(\mathcal{L}_{T_{2}}^{q}\right)$. In particular, for any tree $T$ with $n$ vertices, we have $\lambda_{\max }\left(\mathcal{L}_{P_{n}}^{q}\right) \leq \lambda_{\max }\left(\mathcal{L}_{T}^{q}\right) \leq \lambda_{\max }\left(\mathcal{L}_{S_{n}}^{q}\right)$.

Proof: Assume to the contrary that $\lambda_{\max }\left(\mathcal{L}_{T_{1}}^{q}\right)>\lambda_{\max }\left(\mathcal{L}_{T_{2}}^{q}\right)$. When $x=\lambda_{\max }\left(\mathcal{L}_{T_{1}}^{q}\right)$, by using Remark 25 and (12), we get $\mathcal{D}_{T_{2}}^{T_{1}}\left(q, \lambda_{\max }\left(\mathcal{L}_{T_{1}}^{q}\right)\right)=-f^{\mathcal{L}_{T_{2}}^{q}}\left(q, \lambda_{\max }\left(\mathcal{L}_{T_{1}}^{q}\right)\right)<0$. This contradicts Lemma 24. Thus, $\lambda_{\max }\left(\mathcal{L}_{T_{1}}^{q}\right) \leq \lambda_{\max }\left(\mathcal{L}_{T_{2}}^{q}\right)$ for all $q \in \mathbb{R} \backslash\{0\}$. Using Lemma 4 completes the proof.

In Example 27, we determine all the eigenvalues of $\mathcal{L}_{S_{n}}^{q}$ for all $q \in \mathbb{R}$. Therefore, by Theorem 26. we obtain an upper bound on the largest eigenvalue of $\mathcal{L}_{T}^{q}$ for all $q \in \mathbb{R}$.

Example 27 Let $S_{n}$ be the star tree on the vertex set $[n]$ with $q$-Laplacian $\mathcal{L}_{S_{n}}^{q}$. Without loss of generality in $S_{n}$, we can assume that the vertex 1 has degree $n-1$. We see that the only permutations $(1, j) \in \mathfrak{S}_{n}$ contribute to $\operatorname{det}\left(x I-\mathcal{L}_{S_{n}}^{q}\right)$, where $j=1,2, \ldots, n$. The identity permutation contributes $(x-1)^{n-1}\left(x-1-(n-2) q^{2}\right)$ and each of the remaining $n-1$ transpositions contribute $-q^{2}(x-1)^{n-2}$. Therefore,

$$
\begin{aligned}
f^{\mathcal{L}_{S_{n}}^{q}}(q, x) & =\left[(x-1)^{n-1}\left(x-1-(n-2) q^{2}\right)\right]-\left[(n-1) q^{2}(x-1)^{n-2}\right] \\
& =(x-1)^{n-2}\left[x^{2}-\left(2+(n-2) q^{2}\right) x-q^{2}+1\right] .
\end{aligned}
$$

Thus, the eigenvalues of $\mathcal{L}_{S_{n}}^{q}$ are 1 with multiplicity $n-2$ and $\frac{2+(n-2) q^{2} \pm \sqrt{n^{2} q^{4}+4(n-1)\left(1-q^{2}\right) q^{2}}}{2}$.

The following corollary is an immediate consequence of Theorem 26 . 
Corollary 28 Let $T$ be a tree on $n>1$ vertices with $q$-Laplacian $\mathcal{L}_{T}^{q}$. Then, for all $q \in \mathbb{R}$

$$
\lambda_{\max }\left(\mathcal{L}_{T}^{q}\right) \leq \frac{2+(n-2) q^{2}+\sqrt{n^{2} q^{4}+4(n-1)\left(1-q^{2}\right) q^{2}}}{2} .
$$

\section{2 $\quad \lambda_{\min }\left(\mathcal{L}_{T}^{q}\right)$}

Now we prove a part of Theorem 2 about the smallest eigenvalue of $\mathcal{L}_{T}^{q}$ when $|q| \leq 1$. The proof of the following theorem is very similar to the proof of Theorem 26.

Theorem 29 Let $T_{1}$ and $T_{2}$ be two trees with $n$ vertices such that $T_{2}$ covers $T_{1}$ in $\mathrm{GTS}_{n}$. Then, for all $q \in \mathbb{R} \backslash\{0\}$ with $|q| \leq 1$, we have $\lambda_{\min }\left(\mathcal{L}_{T_{1}}^{q}\right) \geq \lambda_{\min }\left(\mathcal{L}_{T_{2}}^{q}\right)$.

Proof: Assume to the contrary that $\lambda_{\min }\left(\mathcal{L}_{T_{1}}^{q}\right)<\lambda_{\min }\left(\mathcal{L}_{T_{2}}^{q}\right)$. It is easy to see that in this case $q \neq \pm 1$. Therefore from Lemma 5, $0<\lambda_{\min }\left(\mathcal{L}_{T_{1}}^{q}\right)<\lambda_{\min }\left(\mathcal{L}_{T_{2}}^{q}\right)$. By Remark 25, we see that $-f^{\mathcal{L}_{T_{2}}^{q}}\left(q, \lambda_{\min }\left(\mathcal{L}_{T_{1}}^{q}\right)\right)$ is negative if $n$ is even and positive if $n$ is odd. Therefore by (12), $\mathcal{D}_{T_{2}}^{T_{1}}\left(q, \lambda_{\min }\left(\mathcal{L}_{T_{1}}^{q}\right)\right)=-f^{\mathcal{L}_{T_{2}}^{q}}\left(q, \lambda_{\min }\left(\mathcal{L}_{T_{1}}^{q}\right)\right)$ is negative if $n$ is even and positive if $n$ is odd which contradicts Lemma 23 . Thus, $\lambda_{\min }\left(\mathcal{L}_{T_{1}}^{q}\right) \geq \lambda_{\min }\left(\mathcal{L}_{T_{2}}^{q}\right) \geq 0$ and the proof is complete.

We next show that going up on $\mathrm{GTS}_{n}$ decreases the smallest eigenvalue of $\mathcal{L}_{T}^{q}$ when $q \in \mathbb{R}$ with $|q|>1$. The proof of the following theorem is very similar to the proof of Theorem 29 .

Theorem 30 Let $T_{1}$ and $T_{2}$ be two trees on $n$ vertices such that $T_{2}$ covers $T_{1}$ in $\mathrm{GTS}_{n}$. Then, for all $q \in \mathbb{R}$ with $|q|>1$, we have $\lambda_{\min }\left(\mathcal{L}_{T_{1}}^{q}\right) \geq \lambda_{\min }\left(\mathcal{L}_{T_{2}}^{q}\right)$.

Proof: When $q \in \mathbb{R}$ with $|q|>1$, from Lemma 5, $\lambda_{\min }\left(\mathcal{L}_{T_{1}}^{q}\right)<0$ and $\lambda_{\min }\left(\mathcal{L}_{T_{2}}^{q}\right)<0$. Assume to the contrary that $\lambda_{\min }\left(\mathcal{L}_{T_{1}}^{q}\right)<\lambda_{\min }\left(\mathcal{L}_{T_{2}}^{q}\right)<0$. By Remark 25, $f^{\mathcal{L}_{T_{2}}^{q}}\left(q, \lambda_{\text {min }}\left(\mathcal{L}_{T_{1}}^{q}\right)\right)$ is positive when $n$ is even and negative when $n$ is odd. Therefore, by $(12), \mathcal{D}_{T_{2}}^{T_{1}}\left(q, \lambda_{\text {min }}\left(\mathcal{L}_{T_{1}}^{q}\right)\right)=$ $-f^{\mathcal{L}_{T_{2}}^{q}}\left(q, \lambda_{\min }\left(\mathcal{L}_{T_{1}}^{q}\right)\right)$ is negative when $n$ is even and positive when $n$ is odd. This contradicts Lemma 23. Thus, $0>\lambda_{\min }\left(\mathcal{L}_{T_{1}}^{q}\right) \geq \lambda_{\min }\left(\mathcal{L}_{T_{2}}^{q}\right)$, completing the proof.

\section{3 $\lambda_{a}\left(\mathcal{L}_{T}^{q}\right)$}

We next show that as we go up on $\mathrm{GTS}_{n}$, the second smallest eigenvalue of $\mathcal{L}_{T}^{q}$ increases for all $q \in \mathbb{R}$. We begin with the following.

Remark 31 Let $T_{1}$ and $T_{2}$ be two trees on $n$ vertices such that $T_{2}$ covers $T_{1}$ in $\mathrm{GTS}_{n}$. By the interlacing theorem, the smallest eigenvalue of $\mathcal{L}_{T_{i}}^{q} \mid\{1, k\}$ lies between $\lambda_{\min }\left(\mathcal{L}_{T_{i}}^{q}\right)$ and $\lambda_{n-2}\left(\mathcal{L}_{T_{i}}^{q}\right)$, where $i=1,2$.

From Lemma 7, we recall that for all $q \in \mathbb{R} \backslash\{0\}$, the algebraic multiplicity of $\lambda_{\min }\left(\mathcal{L}_{T}^{q}\right)$ as an eigenvalue of $\mathcal{L}_{T}^{q}$ is 1 . This is required to prove the following result.

Lemma 32 Let $T_{1}$ and $T_{2}$ be two trees on $n$ vertices such that $T_{2}$ covers $T_{1}$ in $\mathrm{GTS}_{n}$. Then, for all $q \in \mathbb{R} \backslash\{0\}$, we have $\lambda_{\min }\left(\mathcal{L}_{T_{1}}^{q}\right) \leq \lambda_{a}\left(\mathcal{L}_{T_{2}}^{q}\right)$. 
Proof: For a fixed $q \in \mathbb{R}$ with $|q| \geq 1$, From Lemma 5 , we get $\lambda_{\min }\left(\mathcal{L}_{T_{1}}^{q}\right) \leq 0<\lambda_{a}\left(\mathcal{L}_{T_{2}}^{q}\right)$. When $q \in \mathbb{R} \backslash\{0\}$ with $|q|<1$, assume to the contrary that $\lambda_{\min }\left(\mathcal{L}_{T_{1}}^{q}\right)>\lambda_{a}\left(\mathcal{L}_{T_{2}}^{q}\right)$. Therefore by Lemma 7, $\lambda_{a}\left(\mathcal{L}_{T_{1}}^{q}\right)>\lambda_{\min }\left(\mathcal{L}_{T_{1}}^{q}\right)>\lambda_{a}\left(\mathcal{L}_{T_{2}}^{q}\right)>\lambda_{\min }\left(\mathcal{L}_{T_{2}}^{q}\right)>0$. From $(12), \mathcal{D}_{T_{2}}^{T_{1}}\left(q, \lambda_{\min }\left(\mathcal{L}_{T_{1}}^{q}\right)\right)=$ $-f^{\mathcal{L}_{T_{2}}^{q}}\left(q, \lambda_{\min }\left(\mathcal{L}_{T_{1}}^{q}\right)\right)$. Thus, by Lemma $23 . f^{\mathcal{L}_{T_{2}}^{q}}\left(q, \lambda_{\min }\left(\mathcal{L}_{T_{1}}^{q}\right)\right)$ is negative if $n$ is even and positive if $n$ is odd. On the other hand by Remark 25 and by the IVT, either $f^{\mathcal{L}_{T_{2}}^{q}}(q, x)$ evaluates to a positive quantity if $n$ is even and negative quantity if $n$ is odd for some $x \in\left(\lambda_{a}\left(\mathcal{L}_{T_{2}}^{q}\right), \lambda_{\min }\left(\mathcal{L}_{T_{1}}^{q}\right)\right)$ or there is an eigenvalue $\lambda_{n-2}\left(\mathcal{L}_{T_{2}}^{q}\right)$ of $\mathcal{L}_{T_{2}}^{q}$ in the interval $\left[\lambda_{a}\left(\mathcal{L}_{T_{2}}^{q}\right), \lambda_{\min }\left(\mathcal{L}_{T_{1}}^{q}\right)\right)$. Thus, in both the cases, there must be an eigenvalue $\lambda_{n-2}\left(\mathcal{L}_{T_{2}}^{q}\right)$ of $\mathcal{L}_{T_{2}}^{q}$ in the interval $\left[\lambda_{a}\left(\mathcal{L}_{T_{2}}^{q}\right), \lambda_{\text {min }}\left(\mathcal{L}_{T_{1}}^{q}\right)\right)$ such that

$$
\lambda_{a}\left(\mathcal{L}_{T_{1}}^{q}\right)>\lambda_{\min }\left(\mathcal{L}_{T_{1}}^{q}\right)>\lambda_{n-2}\left(\mathcal{L}_{T_{2}}^{q}\right) \geq \lambda_{a}\left(\mathcal{L}_{T_{2}}^{q}\right)>\lambda_{\min }\left(\mathcal{L}_{T_{2}}^{q}\right)
$$

which contradicts Remark 31 and hence the proof is complete.

The proof of the following theorem is very similar to the proof of Theorem 26 .

Theorem 33 Let $T_{1}$ and $T_{2}$ be two trees with $n$ vertices such that $T_{2}$ covers $T_{1}$ in $\mathrm{GTS}_{n}$. Then, for all $q \in \mathbb{R} \backslash\{0\}$, we have $\lambda_{a}\left(\mathcal{L}_{T_{1}}^{q}\right) \leq \lambda_{a}\left(\mathcal{L}_{T_{2}}^{q}\right)$. In particular, for any tree $T$ with $n$ vertices, $\lambda_{a}\left(\mathcal{L}_{P_{n}}^{q}\right) \leq \lambda_{a}\left(\mathcal{L}_{T}^{q}\right) \leq \lambda_{a}\left(\mathcal{L}_{S_{n}}^{q}\right)$.

Proof: $\quad$ Assume to the contrary that $\lambda_{a}\left(\mathcal{L}_{T_{1}}^{q}\right)>\lambda_{a}\left(\mathcal{L}_{T_{2}}^{q}\right)>0$. From Lemma 32, $\lambda_{a}\left(\mathcal{L}_{T_{1}}^{q}\right)>$ $\lambda_{a}\left(\mathcal{L}_{T_{2}}^{q}\right) \geq \max \left(0, \lambda_{\min }\left(\mathcal{L}_{T_{1}}^{q}\right)\right)$. By Remark 25, $f^{\mathcal{L}_{T_{1}}^{q}}\left(q, \lambda_{a}\left(\mathcal{L}_{T_{2}}^{q}\right)\right)$ is non-positive when $n$ is even and non-negative when $n$ is odd. Thus, from $(12), \mathcal{D}_{T_{2}}^{T_{1}}\left(q, \lambda_{a}\left(\mathcal{L}_{T_{2}}^{q}\right)\right)=f^{\mathcal{L}_{T_{1}}^{q}}\left(q, \lambda_{a}\left(\mathcal{L}_{T_{2}}^{q}\right)\right)$ is nonpositive when $n$ is even and non-negative when $n$ is odd. This contradicts Lemma 23. By Lemma 4, the proof is complete.

From Example 27, when $n>2$ we get $\lambda_{a}\left(\mathcal{L}_{S_{n}}^{q}\right)=1$. Thus, we obtain the following corollary.

Corollary 34 Let $T$ be a tree on $n>2$ vertices with $q$-Laplacian $\mathcal{L}_{T}^{q}$. Then, for all $q \in \mathbb{R}$, we have $\lambda_{a}\left(\mathcal{L}_{T}^{q}\right) \leq 1$.

The following example illustrates Theorem 2

Example 35 Let $T_{1}$ and $T_{2}$ be the two trees given in Figure 4 Clearly $T_{2}$ covers $T_{1}$ in $\mathrm{GTS}_{6}$. Let $\mathcal{L}_{T_{1}}^{q}$ and $\mathcal{L}_{T_{2}}^{q}$ be the $q$-Laplacians of $T_{1}$ and $T_{2}$ respectively. When $q \in\{0.1,0.5,1.0,1.5,10\}$, the largest, the smallest and the second smallest eigenvalues of $\mathcal{L}_{T_{i}}^{q}$ are given in Table 1 , where $i=1$, 2. Calculations were done by using the computer package SageMath.
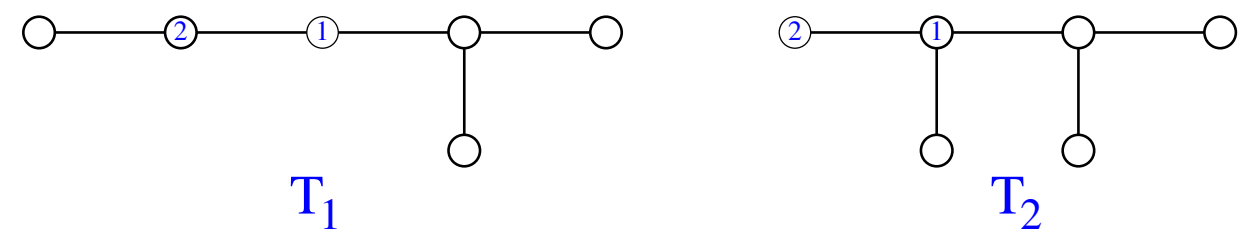

Figure 4: An example of $\mathrm{GTS}_{6}$ 


\begin{tabular}{|r||r|r||r||r||r|r|}
\hline \multicolumn{1}{|||}{} & \multicolumn{2}{|c||}{$\lambda_{\max }\left(\mathcal{L}_{T_{i}}^{q}\right)$} & \multicolumn{2}{c||}{$\lambda_{\min }\left(\mathcal{L}_{T_{i}}^{q}\right)$} & \multicolumn{2}{c|}{$\lambda_{a}\left(\mathcal{L}_{T_{i}}^{q}\right)$} \\
\hline$q$ & $\lambda_{\max }\left(\mathcal{L}_{T_{1}}^{q}\right)$ & $\lambda_{\max }\left(\mathcal{L}_{T_{2}}^{q}\right)$ & $\lambda_{\min }\left(\mathcal{L}_{T_{1}}^{q}\right)$ & $\lambda_{\min }\left(\mathcal{L}_{T_{2}}^{q}\right)$ & $\lambda_{a}\left(\mathcal{L}_{T_{1}}^{q}\right)$ & $\lambda_{a}\left(\mathcal{L}_{T_{2}}^{q}\right)$ \\
\hline \hline 0.1 & 1.2017 & 1.2136 & 0.8208 & 0.8130 & 0.8890 & 0.9064 \\
\hline 0.5 & 2.2566 & 2.3660 & 0.3032 & 0.2929 & 0.5586 & 0.6340 \\
\hline 1.0 & 4.2143 & 4.5616 & 0.0000 & 0.0000 & 0.3249 & 0.4384 \\
\hline 1.5 & 6.9857 & 7.6742 & -0.0864 & -0.0981 & 0.2014 & 0.3258 \\
\hline 10.0 & 202.9803 & 211.9481 & -0.0069 & -0.0469 & 0.0070 & 0.0519 \\
\hline
\end{tabular}

Table 1: The eigenvalues $\lambda_{\max }\left(\mathcal{L}_{T_{i}}^{q}\right), \lambda_{\min }\left(\mathcal{L}_{T_{i}}^{q}\right)$ and $\lambda_{a}\left(\mathcal{L}_{T_{i}}^{q}\right)$

\section{Eigenvalues of the $q, t$-Laplacian}

Let $T$ be a tree with $n$ vertices. A generalization $\mathcal{L}_{T}^{q, t}$ was defined by Bapat and Sivasubramanian in [2]. Orient the edges of $T$ arbitrarily. Since $T$ is now directed, we use directed graph terminology for its arcs $(i, j)$. Define $\mathcal{L}_{T}^{q, t}=\left(\ell_{i, j}\right)_{1 \leq i, j \leq n}$ by $\ell_{i, j}=-q$, if $\{i, j\}$ is an edge $e$ in $T$ and the orientation of $e$ gives the arc $(i, j)$. If the orientation of $e=\{i, j\}$ gives the $\operatorname{arc}(i, j)$, then, define $\ell_{j, i}=-t$. If $\{i, j\}=e$ with $i \neq j$ is not an edge in $T$, then, define $\ell_{i, j}=0$. Define $\ell_{i, i}=1+q t\left(d_{i}-1\right)$, where $d_{i}$ is the degree of the $i$-th vertex in $T$. It is easy to see that when $q=t$, $\mathcal{L}_{T}^{q, t}=\mathcal{L}_{T}^{q}$. Thus, $\mathcal{L}_{T}^{q, t}$ is a generalization of $\mathcal{L}_{T}^{q}$.

Let $T$ be a tree on $n$ vertices with $q, t$-Laplacian $\mathcal{L}_{T}^{q, t}$. For fixed but arbitrary $q, t \in \mathbb{C}$, let $f^{\mathcal{L}_{T}^{q, t}}(q, t, x)$ be the characteristic polynomial of $\mathcal{L}_{T}^{q, t}$ in three variables $q, t$ and $x$. Clearly Lemma 10 and Theorem 12 go through for $f^{\mathcal{L}_{T}^{q, t}}(q, t, x)$ when $q \neq 0$ and $t \neq 0$. In this case, it is easy to see that $y_{1}=0, y_{2}=1, y_{3}=-(x-1+q t)$ and $y_{4}=-1 / q t x$.

We need the Interlacing Theorem for eigenvalues of Hermitian matrices to generalize Lemmas 19 and 20 to $\mathcal{L}_{T}^{q, t}$. Hence, we require $\mathcal{L}_{T}^{q, t}$ to be Hermitian. We see that the matrix $\mathcal{L}_{T}^{q, t}$ is Hermitian for all $q, t \in \mathbb{C}$ with $\bar{q}=t$. When $\bar{q}=t$, the proof of the $q, t$-version of the general lemma is identical to that of Lemma 10. Bapat and Sivasubramanian [2] proved that $\operatorname{det}\left(\mathcal{L}_{T}^{q, t}\right)=1-q t$. For $v \in T$, Nagar and Sivasubramanian [12] proved that $f^{\mathcal{L}_{T}^{q, t} \mid v}(q, t, 0)=(-1)^{n-1}$ for all $q, t \in \mathbb{C}$. When $\bar{q}=t$ in $\mathbb{C} \backslash\{0\}$, we get $q t=|q|^{2}>0$ and thus, Lemmas 19 and 20 go through for $\mathcal{L}_{T}^{q, t}$.

When $\bar{q}=t \in \mathbb{C}$, Theorem 2 also goes through for the bivariate Laplacian matrix $\mathcal{L}_{T}^{q, t}$. Let $\lambda_{\min }\left(\mathcal{L}_{T}^{q, t}\right), \lambda_{a}\left(\mathcal{L}_{T}^{q, t}\right)$ and $\lambda_{\max }\left(\mathcal{L}_{T}^{q, t}\right)$ be the smallest, the second smallest and the largest eigenvalues of $\mathcal{L}_{T}^{q, t}$ respectively. We get the following result as a generalization of Theorem 2. As its proof is very similar to the proofs of Theorems 26, 29, 30 and 33, we omit and merely state the result.

Theorem 36 Let $T_{1}$ and $T_{2}$ be two trees on $n$ vertices such that $T_{2}$ covers $T_{1}$ in $\mathrm{GTS}_{n}$. Let $\mathcal{L}_{T_{1}}^{q, t}$ and $\mathcal{L}_{T_{2}}^{q, t}$ be the bivariate Laplacians of $T_{1}$ and $T_{2}$ respectively. Then, for $q, t \in \mathbb{C}$ with $\bar{q}=t$, we have $\lambda_{\min }\left(\mathcal{L}_{T_{1}}^{q, t}\right) \geq \lambda_{\min }\left(\mathcal{L}_{T_{2}}^{q, t}\right), \lambda_{\max }\left(\mathcal{L}_{T_{1}}^{q, t}\right) \leq \lambda_{\max }\left(\mathcal{L}_{T_{2}}^{q, t}\right)$ and $\lambda_{a}\left(\mathcal{L}_{T_{1}}^{q, t}\right) \leq \lambda_{a}\left(\mathcal{L}_{T_{2}}^{q, t}\right)$.

Thus, for all $\bar{q}=t \in \mathbb{C}$, three eigenvalues of $\mathcal{L}_{T}^{q, t}$ exhibit monotonicity when we go up on $\mathrm{GTS}_{n}$. Moreover, for the largest and second smallest eigenvalues max-min pair is $\left(S_{n}, P_{n}\right)$ while for the smallest eigenvalue max-min pair is $\left(P_{n}, S_{n}\right)$. It is easy to determine the eigenvalues of $\mathcal{L}_{S_{n}}^{q, t}$ as done in Example 27. Thus, we get the following corollary of Theorem 36. 
Corollary 37 Let $T$ be a tree on $n>2$ vertices with $q$, $t$-Laplacian $\mathcal{L}_{T}^{q, t}$. Then, for all $q, t \in \mathbb{C}$ with $\bar{q}=t$, we have $\lambda_{a}\left(\mathcal{L}_{T_{1}}^{q, t}\right) \leq 1$ and

$$
\lambda_{\max }\left(\mathcal{L}_{T}^{q, t}\right) \leq \frac{2+(n-2) q t+\sqrt{n^{2} q^{2} t^{2}+4(n-1)(1-q t) q t}}{2} .
$$

When $q=1 / t \in \mathbb{C}$ with $t \neq 0$ and $\bar{q} \neq t$, the matrix $\mathcal{L}_{T}^{q, t}$ is no longer Hermitian but our result follows from Nagar and Sivasubramanian [12, Remark 34]. There it was proved that when $q=1 / t \in \mathbb{C}$ with $t \neq 0, \operatorname{det}\left(x I-\mathcal{L}_{T}^{q, t}\right)=\operatorname{det}\left(x I-L_{T}\right)$, where $L_{T}$ is the Laplacian matrix of $T$. Thus, we have $\lambda_{\min }\left(\mathcal{L}_{T}^{q, t}\right)=\lambda_{\min }\left(L_{T}\right), \lambda_{\max }\left(\mathcal{L}_{T}^{q, t}\right)=\lambda_{\max }\left(L_{T}\right)$ and $\lambda_{a}\left(\mathcal{L}_{T}^{q, t}\right)=\lambda_{a}\left(L_{T}\right)$. Moreover, we have $\lambda_{\max }\left(\mathcal{L}_{T}^{q, t}\right)=\lambda_{\max }\left(L_{T}\right) \leq n$ when $q=1 / t \in \mathbb{C}$ with $t \neq 0$.

One special case of $\mathcal{L}_{T}^{q, t}$ is obtained when we set $q=\imath$ and $t=-\imath$, where $\imath=\sqrt{-1}$. In this case, the matrix $\mathcal{L}_{T}^{q, t}$ is the Hermitian positive semidefinite (see Bapat and Sivasubramanian [2]). The bivariate Laplacian matrix $\mathcal{L}_{T}^{q, t}$ is called the Hermitian Laplacian matrix of $T$ when $q=\imath$ and $t=-\imath$ defined by $\mathrm{Yu}$ and $\mathrm{Qu}$ [14]. In this case, we have both $\bar{q}=t$ and $q=1 / t$.

\section{Eigenvalue monotonicity of $E D_{T}$}

Let $T$ be a tree on $n$ vertices with exponential distance matrix $\mathrm{ED}_{T}$. Let the eigenvalues of $\mathrm{ED}_{T}$ be $\lambda_{\max }\left(\mathrm{ED}_{T}\right)=\lambda_{1}\left(\mathrm{ED}_{T}\right) \geq \lambda_{2}\left(\mathrm{ED}_{T}\right) \geq \cdots \geq \lambda_{n}\left(\mathrm{ED}_{T}\right)=\lambda_{\min }\left(\mathrm{ED}_{T}\right)$. When $q= \pm 1$, it is simple to see that the eigenvalues of $\mathrm{ED}_{T}$ are $n$ and 0 with multiplicities 1 and $n-1$ respectively. Thus, in this case, the eigenvalues of $\mathrm{ED}_{T}$ are constant on $\mathrm{GTS}_{n}$. By Lemmas 5 and 6, we see that $\mathrm{ED}_{T}$ is a positive definite matrix when $q \in \mathbb{R}$ with $|q|<1$ and $\mathrm{ED}_{T}$ has exactly one positive eigenvalue when $q \in \mathbb{R}$ with $|q|>1$. Let $\lambda_{\min }\left(\mathrm{ED}_{T}\right), \lambda_{\max }\left(\mathrm{ED}_{T}\right)$ and $\lambda_{2}\left(\mathrm{ED}_{T}\right)$ be the smallest, the largest and the second largest eigenvalues of $\mathrm{ED}_{T}$. The following theorem is our main result of this section.

Theorem 38 Let $T_{1}$ and $T_{2}$ be two trees with $n$ vertices such that $T_{2}$ covers $T_{1}$ in $\mathrm{GTS}_{n}$.

1. If $q \in \mathbb{R}$ with $|q|<1$, then, $\lambda_{\min }\left(\mathrm{ED}_{T_{1}}\right) \geq \lambda_{\min }\left(\mathrm{ED}_{T_{2}}\right), \lambda_{2}\left(\mathrm{ED}_{T_{1}}\right) \geq \lambda_{2}\left(\mathrm{ED}_{T_{2}}\right)$ and $\lambda_{\max }\left(\mathrm{ED}_{T_{1}}\right) \leq \lambda_{\max }\left(\mathrm{ED}_{T_{2}}\right)$.

2. If $q \in \mathbb{R}$ with $|q|>1$, then, $\lambda_{\min }\left(\mathrm{ED}_{T_{1}}\right) \leq \lambda_{\min }\left(\mathrm{ED}_{T_{2}}\right), \lambda_{2}\left(\mathrm{ED}_{T_{1}}\right) \leq \lambda_{2}\left(\mathrm{ED}_{T_{2}}\right)$ and $\lambda_{\max }\left(\mathrm{ED}_{T_{1}}\right) \geq \lambda_{\max }\left(\mathrm{ED}_{T_{2}}\right)$.

Proof: When $q \in \mathbb{R}$ with $|q|<1$, by using Lemma 6, all the eigenvalues of $\mathrm{ED}_{T_{1}}$ and $\mathrm{ED}_{T_{2}}$ are positive and they are

$$
\begin{aligned}
\lambda_{\max }\left(\mathrm{ED}_{T_{1}}\right) & =\frac{1-q^{2}}{\lambda_{\min }\left(\mathcal{L}_{T_{1}}^{q}\right)} \geq \frac{1-q^{2}}{\lambda_{a}\left(\mathcal{L}_{T_{1}}^{q}\right)} \geq \cdots \geq \frac{1-q^{2}}{\lambda_{1}\left(\mathcal{L}_{T_{1}}^{q}\right)}=\frac{1-q^{2}}{\lambda_{\max }\left(\mathcal{L}_{T_{1}}^{q}\right)} \\
\text { and } \quad \lambda_{\max }\left(\mathrm{ED}_{T_{2}}\right) & =\frac{1-q^{2}}{\lambda_{\min }\left(\mathcal{L}_{T_{2}}^{q}\right)} \geq \frac{1-q^{2}}{\lambda_{a}\left(\mathcal{L}_{T_{2}}^{q}\right)} \geq \cdots \geq \frac{1-q^{2}}{\lambda_{1}\left(\mathcal{L}_{T_{2}}^{q}\right)}=\frac{1-q^{2}}{\lambda_{\max }\left(\mathcal{L}_{T_{2}}^{q}\right)}
\end{aligned}
$$


respectively. Similarly, when $|q|>1$, by Lemma 6 , both the matrices $\mathrm{ED}_{T_{1}}$ and $\mathrm{ED}_{T_{2}}$ have exactly one positive eigenvalue and their eigenvalues are

$$
\begin{aligned}
\lambda_{\max }\left(\mathrm{ED}_{T_{1}}\right) & =\frac{1-q^{2}}{\lambda_{\min }\left(\mathcal{L}_{T_{1}}^{q}\right)} \geq \frac{1-q^{2}}{\lambda_{\max }\left(\mathcal{L}_{T_{1}}^{q}\right)} \geq \cdots \geq \frac{1-q^{2}}{\lambda_{n-1}\left(\mathcal{L}_{T_{1}}^{q}\right)}=\frac{1-q^{2}}{\lambda_{a}\left(\mathcal{L}_{T_{1}}^{q}\right)} \\
\text { and } \quad \lambda_{\max }\left(\mathrm{ED}_{T_{2}}\right) & =\frac{1-q^{2}}{\lambda_{\min }\left(\mathcal{L}_{T_{2}}^{q}\right)} \geq \frac{1-q^{2}}{\lambda_{\max }\left(\mathcal{L}_{T_{2}}^{q}\right)} \geq \cdots \geq \frac{1-q^{2}}{\lambda_{n-1}\left(\mathcal{L}_{T_{2}}^{q}\right)}=\frac{1-q^{2}}{\lambda_{a}\left(\mathcal{L}_{T_{2}}^{q}\right)}
\end{aligned}
$$

respectively. Thus, by using (13), (14) and Theorem 2 the proof is complete.

Thus, for all $q \in \mathbb{R}$, three eigenvalues of $\mathrm{ED}_{T}$ exhibit monotonicity when we go up on $\mathrm{GTS}_{n}$ and max-min pair is either $\left(P_{n}, S_{n}\right)$ or $\left(S_{n}, P_{n}\right)$.

\section{1 $q, t$-exponential distance matrix}

We consider the bivariate exponential distance matrix $\mathrm{ED}_{T}^{q, t}$ of a tree $T$. Orient the tree $T$ as done in Section 7. Thus each directed arc $e$ of $E(T)$ has a unique reverse arc $e_{\text {rev }}$ and we assign a variable weight $w(e)=q$ and $w\left(e_{\text {rev }}\right)=t$ or vice versa. If the path $P_{i, j}$ from vertex $i$ to vertex $j$ has the sequence of edges $P_{i, j}=\left(e_{1}, e_{2}, \ldots, e_{p}\right)$, assign it weight $w_{i, j}=\prod_{e_{k} \in P_{i, j}} w\left(e_{k}\right)$. Define $w_{i, i}=1$ for $i=1,2, \ldots, n$. Define the bivariate $q, t$-exponential distance matrix $\operatorname{ED}_{T}^{q, t}=\left(w_{i, j}\right)_{1 \leq i, j \leq n}$. Clearly, when $q=t$, we will have $\mathrm{ED}_{T}^{q, t}=\mathrm{ED}_{T}$. Bapat and Sivasubramanian in [2, Theorem 3.2] showed the following bivariate counterpart of Lemma 6 .

Lemma 39 (Bapat and Sivasubramanian) Let $T$ be a tree on $n$ vertices with $q, t$-Laplacian $\mathcal{L}_{T}^{q, t}$ and $q$, t-exponential distance matrix $\mathrm{ED}_{T}^{q, t}$. When $q t \neq 1$, then $\left(\mathrm{ED}_{T}^{q, t}\right)^{-1}=\frac{1}{1-q t} \mathcal{L}_{T}^{q, t}$. Moreover, $\frac{1-q t}{\lambda_{i}\left(\mathcal{L}_{T}^{q, t}\right)}$ is an eigenvalue of $\mathrm{ED}_{T}^{q, t}$, where $\lambda_{i}\left(\mathcal{L}_{T}^{q, t}\right)$ is an eigenvalue of $\mathcal{L}_{T}^{q, t}$.

From Theorem 36, it is easy to see that Theorem 38 goes through for the bivariate $q, t$-exponential distance matrix $\mathrm{ED}_{T}^{q, t}$ when $q, t \in \mathbb{C}$ with $\bar{q}=t$ and $q t \neq 1$.

\section{Acknowledgement}

The author acknowledges support from DST, New Delhi for providing a Senior Research Fellowship. Our main theorem in this work was in its conjecture form, tested using the computer package "SageMath". We thank the authors for generously releasing SageMath as an open-source package.

\section{References}

[1] Bapat, R. B., LAL, A. K., And PATi, S. A $q$-analogue of the distance matrix of a tree. Linear Algebra and its Applications 416 (2006), 799-814. 2, 3, 4

[2] Bapat, R. B., and Sivasubramanian, S. The Product Distance Matrix of a Tree and a Bivariate Zeta Function of a Graph. Electronic Journal of Linear Algebra 23 (2012), 275286. 2, 16, 17, 18 
[3] BASs, H. The Ihara-Selberg Zeta Function of a Tree Lattice. International Journal of Math. 3 (1992), 717-797. 2

[4] CSIKVÁRI, P. On a conjecture of V. Nikiforov. Discrete Mathematics 309, 13 (2009), 45224526. 1

[5] Csikvári, P. On a Poset of Trees. Combinatorica 30 (2) (2010), 125-137. 1, 3

[6] CSIKVÁRI, P. On a Poset of Trees II. Journal of Graph Theory 74 (2013), 81-103. 1, 2, 3 , 5, 10

[7] Foata, D., And Zeilberger, D. Combinatorial Proofs of Bass's Evaluations of the IharaSelberg Zeta function of a Graph. Transactions of the AMS 351 (1999), 2257-2274. 2

[8] Godsil, C. D., And Royle, G. Algebraic Graph Theory. Springer-Verlag New York, 2001. 4, 10

[9] Horn, R. A., And Johnson, C. R. Matrix Analysis, 2 ed. Cambridge University Press, 2012. 4

[10] Kelmans, A. K. On Graphs with Randomly Deleted Edges. Acta Mathematica Acad. Sci. Hungarica 37 (1981), 77-88. 1

[11] Molitierno, J. J. Applications of Combinatorial Matrix Theory to Laplacian Matrices of Graphs. CRC Press, 2012. 7

[12] Nagar, M. K., AND Sivasubramanian, S. Laplacian Immanantal polynomials and the GTS poset on trees. Preprint available at arXiv: https://arxiv.org/abs/1710.02416 (2017). 4. 9, 16, 17

[13] Satyanarayana, A., Schoppman, L., And Suffel, C. L. A Reliability-Improving Graph Transformation with Applications to Network eliability. Networks 22 (1992), 209216. 1

[14] YU, G., AND QU, H. Hermitian Laplacian matrix and positive of mixed graphs. Applied Mathematics and Computation 269 (2015), 70-76. 17 